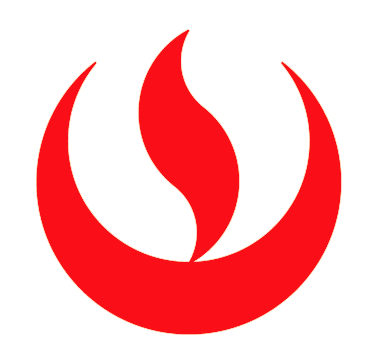

UNIVERSIDAD PERUANA DE CIENCIAS APLICADAS

Facultad De Psicología

Carrera De Psicología

\title{
Satisfacción de Necesidades Psicológicas Básicas y Estilos de Motivación en Supervisores de Empresas en Lima Metropolitana
}

\author{
TESIS \\ Para optar el título profesional de: Licenciada en Psicología
}

AUTOR

Garcia Hoefken, Alexia (0000-0003-4021-8250)

ASESOR DE TESIS

Gargurevich Liberti, Rafael Ernesto (0000-0001-6346-4134) 


\section{Resumen}

Esta investigación tuvo como objetivo identificar la relación que existe entre las necesidades psicológicas básicas de supervisores de empresas en Lima Metropolitana y los estilos de motivación que utilizan. Para responder a la pregunta de investigación, se utilizaron los siguientes dos instrumentos: "Basic Psychological Need Satisfaction and Frustration Scale" (BPNSFS) y "Problems at Work Questionnaire" (PAW). Estos instrumentos fueron aplicados a una muestra de 111 supervisores de empresas de Lima Metropolitana donde $62 \%$ fueron hombres y $38 \%$ mujeres, con una edad promedio de 38.6 años. Al asociar las dos variables, se encontró una correlación positiva y significativa de la sub-escala del estilo de alto apoyo a la autonomía con las tres necesidades psicológicas básicas: autonomía $\left(.29^{* *}, p<.01\right)$, competencia $\left(.30^{* *}, p<.01\right)$ y relación $\left(.35^{* * *}, p<.001\right)$. Se llegó a la conclusión que los supervisores que tienen un estilo de motivación que apoya la autonomía tienen satisfechas las tres necesidades psicológicas básicas. Se encontró que la necesidad de relación fue satisfecha en mayor grado.

Palabras Clave: Estilos de motivación, necesidades psicológicas básicas, supervisores, apoyo a la autonomía 


\begin{abstract}
This research aimed to identify the relationship between the basic psychological needs of the supervisor and their motivational style. To answer the research question, two instruments were used: "Basic Psychological Need Satisfaction and Frustration Scale" (BPNSFS) and "Problems at Work Questionnaire" (PAW), respectively. Both instruments were applied to a sample of 111 business supervisors in Metropolitan Lima, 62\% were men and 38\% were women, with an average age of 38.6 years. When the association between the two variables was made, the subscale of high autonomy support had a positive and significant correlation with the three basic psychological need satisfaction: autonomy $\left(.29^{* *}, p<.01\right)$, competence $\left(.30^{* *}, p<.01\right)$ and relatedness $\left(.35^{* * *}, p<.001\right)$. To sum up, supervisors who have a motivational style that supports autonomy have their three basic psychological needs satisfied. The need for relatedness was satisfied to a greater degree.
\end{abstract}

Keywords: Motivational styles, basic psychological needs, supervisors, autonomy support 


\section{Tabla de Contenidos}

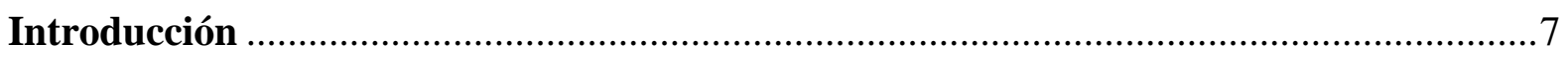

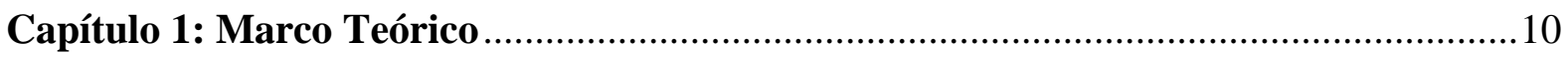

Alcances teóricos de las variables estudiadas ........................................................................ 10

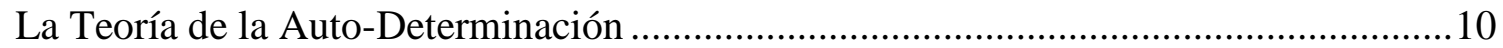

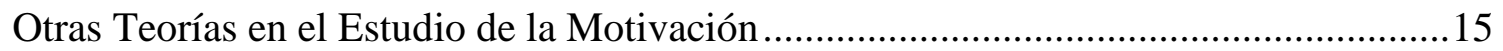

Investigaciones sobre la motivación en Lima Metropolitana .......................................17

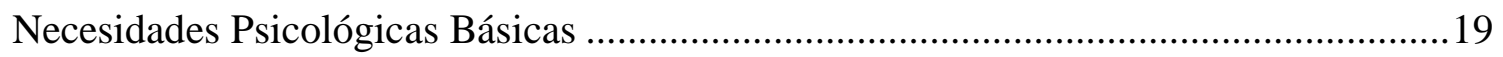

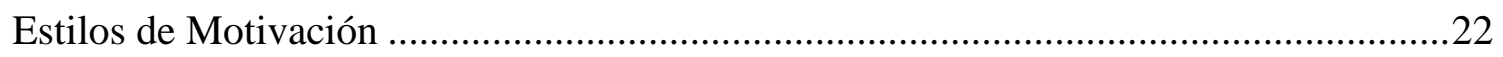

Investigaciones sobre el estilo de motivación y la satisfacción de las necesidades

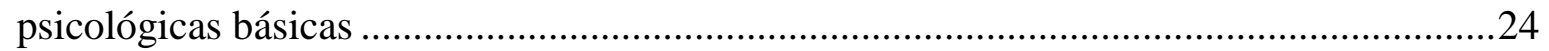

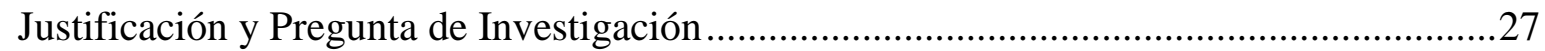

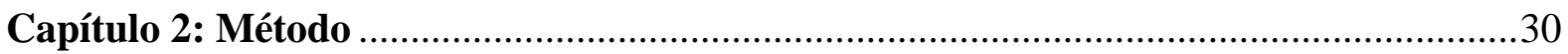

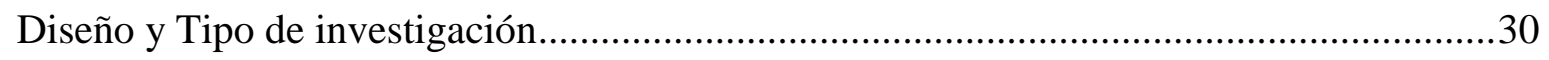

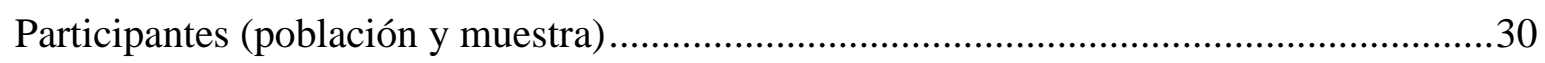

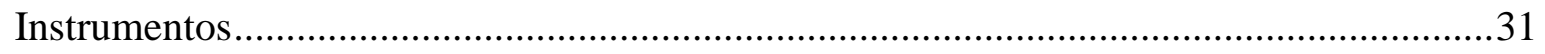

Escala de satisfacción y frustacción de las Necesidades Psicológicas Básicas (BPNSFS)

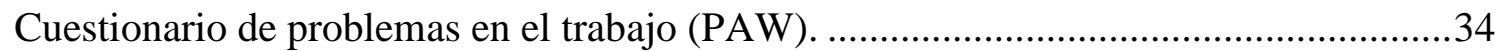

Procedimiento de Recolección y Procesamiento de la Información.....................................38

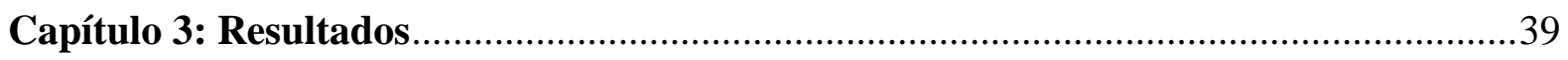

Propiedades Psicométricas de los Instrumentos utilizados: Validez y Confiabilidad .........39

Validez de la Escala de Satisfacción y Frustración de Necesidades Psicológicas Básicas (BPNSFS)

Confiabilidad de la Escala de Satisfacción y Frustración de Necesidades Psicológicas

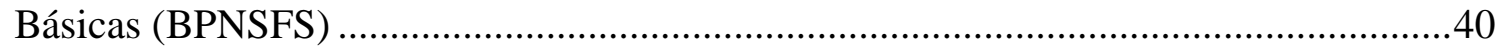

Evidencias de validez del cuestionario problemas en el trabajo (PAW) . ........................42

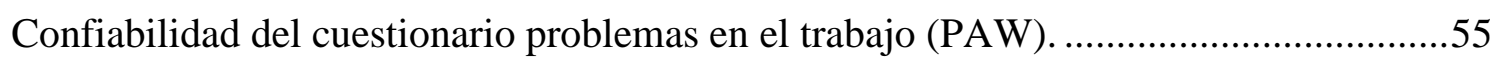

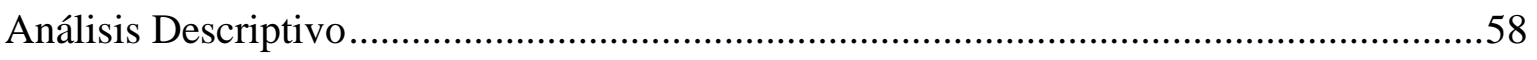

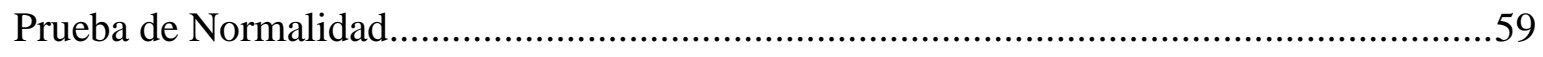

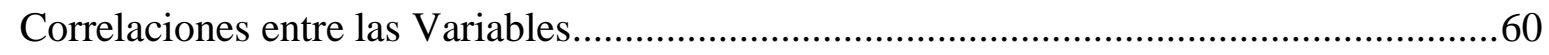




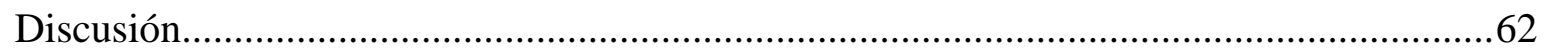

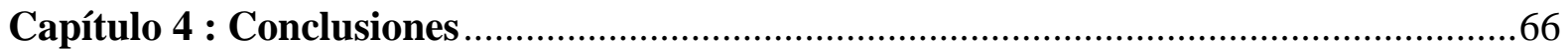

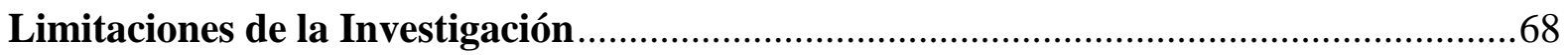

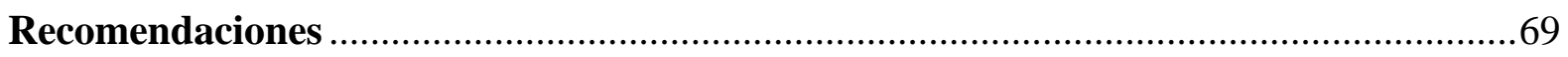

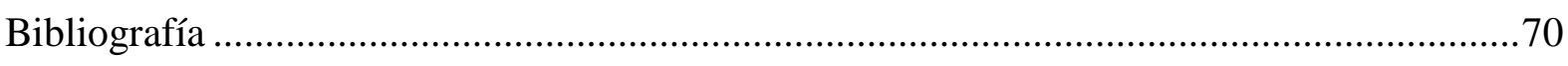

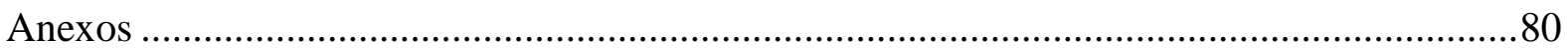




\section{Indice de Tablas}

Tabla 1: Confiabilidad en la Escala de Satisfacción y Frustración de Necesidades Psicológicas

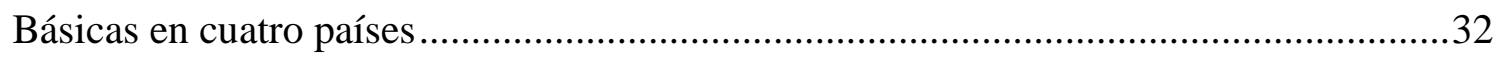

Tabla 2: Autonomía: Correlación Elemento-Total Corregidas.............................................41

Tabla 3: Competencia: Correlación Elemento-Total Corregidas..............................................41

Tabla 4 : Relación: Correlación Elemento-Total Corregidas ..................................................42

Tabla 5 : Matriz de componentes Rotados- Primer análisis .................................................45

Tabla 6 : Matriz de Componentes Rotados - Segundo Análisis............................................48

Tabla 7 : Validez: Matriz de componentes Rotados- Tercer Análisis ...................................51

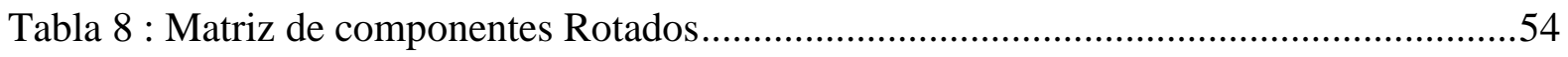

Tabla 9 : Alto Apoyo a la Autonomía: Correlaciones Elemento-Total Corregidas..................56

Tabla 10 : Alto Apoyo al Control: Correlaciones Elemento-Total Corregidas .......................57

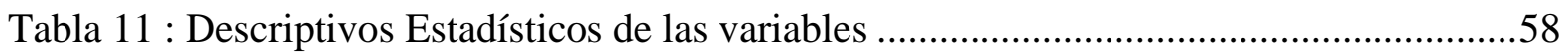

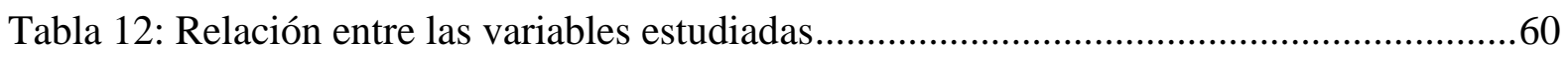




\section{Indice de Gráficos}

Gráfico 1: Gráfico de Sedimentación de Cattel sugiere retener 4 componentes ......................44

Gráfico 2: Gráfico de Sedimentación de Cattel sugiere retener también 4 componentes.......47

Gráfico 3: Gráfico de Sedimentación de Cattel sugiere retener también 2 componentes........51

Gráfico 4: Gráfico de Sedimentación de Cattel sugiere retener también 2 componentes. .......54 


\section{Introducción}

Satisfacción de Necesidades Psicológicas Básicas y Estilos de Motivación en Supervisores de Empresas en Lima Metropolitana

La presente investigación tuvo como objetivo identificar la relación entre el grado de satisfacción de las necesidades psicológicas básicas y los estilos motivación de los supervisores. Esta investigación se desarrolló en el ámbito laboral de empresas en Lima Metropolitana. La población objetivo fueron trabajadores que tenían una persona a su cargo, como mínimo. A continuación, se procederá a describir brevemente las dos variables de esta investigación.

La primera variable es la satisfacción de las necesidades psicológicas básicas. El supervisor, como el resto de personas, tiene tres necesidades psicológicas básicas: necesidad de autonomía, necesidad de sentirse competente y la necesidad de relacionarse con los demás, las cuales pueden ser satisfechas o pueden ser frustradas (Gagné \& Deci, 2005).

Nishimura y Suzuki (2016) realizaron un estudio sobre la satisfacción y frustración de las necesidades psicológicas básicas. La muestra fue de 564 estudiantes de la ciudad de Tokyo y se encontró que la satisfacción de cada una de las tres necesidades psicológicas básicas pudo contribuir en la predicción del bienestar subjetivo (satisfacción en general y vitalidad), mientras que la frustración de cada una de las necesidades logró predecir un malestar (estado de ánimo depresivo). Si bien el estudio fue realizado dentro del ámbito educativo, se puede llegar a la conclusión que la satisfacción de las necesidades psicológicas básicas genera un efecto positivo en el bienestar general de las personas. 
Los estilos motivacionales son la segunda variable de estudio en esta investigación. Según la Teoría de la Auto-Determinación, existen dos estilos utilizados para motivar a las personas, sea a través del apoyo a la autonomía o ejerciendo control. Un estilo motivacional de apoyo a la autonomía se refiere a lograr entender la perspectiva del otro, empoderar a la persona y darle la capacidad de elegir por si misma. El estilo controlador busca motivar a los empleados a través de órdenes, instructivos y castigos, generando sentimientos de vergüenza, ansiedad, culpa, entre otros. El estilo de motivación que un supervisor utilice puede generar un impacto de manera positiva o negativa en el empleado, lo cual influye en su capacidad de desempeño y rendimiento en el trabajo (Deci, Connell, \& Ryan, 1989; Deci \& Ryan, 2008).

Dentro del ambiente laboral, Gillet, Forest, Benabou y Bentein (2015) analizaron las necesidades psicológicas básicas. y la percepción de los estilos motivacionales. Se tomó como muestra 129 estudiantes de MBA que tenían una jornada laboral a tiempo completo. En los resultados se encontró que mientras los empleados percibían más apoyo del líder y menos conflicto de roles en el trabajo, la satisfacción de sus necesidades psicológicas básicas era mayor y menor la frustración de estas. Esta investigación muestra la importancia y beneficios de un estilo motivacional de autonomía y cómo afecta en la satisfacción de necesidades psicológicas básicas de los empleados.

Por consiguiente, esta investigación buscó identificar la relación que existe entre la satisfacción de las necesidades básicas y los estilos motivacionales del supervisor. Cabe mencionar que existen estudios que analizan la percepción que tienen los trabajadores sobre el estilo motivacional del supervisor. Sin embargo, esta investigación se centró en identificar el estilo motivacional del supervisor y la satisfacción de las necesidades psicológicas básicas del supervisor, y se analizó la relación que existe entre ellas. 
Como objetivo específico se propuso establecer el nivel de satisfacción de las necesidades psicológicas básicas de los supervisores e identificar el estilo de motivación que emplea el supervisor en su contexto de trabajo.

Para lograr el objetivo principal de esta investigación se utilizaron dos herramientas. La primera es la Escala de Satisfacción y Frustración de Necesidades Psicológicas Básicas (BPNSFS), la cual se utilizó para medir el grado de satisfacción de las necesidades en los supervisores. La segunda herramienta es el Cuestionario sobre los Problemas en el Trabajo o “The Problems at Work Questionnaire (PAW)", el cual analizó el estilo de motivación que utiliza el supervisor en el ambiente laboral. En otras palabras, la tendencia que tiene el supervisor a motivar apoyando la autonomía y/o ejerciendo control (Deci et al., 1989).

El esquema de la presente investigación inicia con el marco teórico, donde se explica la Teoría de la Auto-Determinación y se describen las variables que se analizarán. En seguida, se plantea la pregunta de investigación, los objetivos y la justificación, donde se argumenta por qué es importante realizar esta investigación. Por último, se definen los métodos a utilizar, se exploran los resultados obtenidos y se realizan conclusiones y limitaciones de la presente investigación. 


\section{Capítulo 1: Marco Teórico}

\section{Alcances teóricos de las variables estudiadas}

\section{La Teoría de la Auto-Determinación}

La Teoría de la Auto-Determinación es una macro teoría de la motivación humana, las emociones y la personalidad, la cual ha estado en desarrollo durante 40 años tras el fundamental trabajo de Edward Deci y Richard Ryan (Vansteenkiste, Niemiec \& Soens, 2010). Esta teoría, postula que el ser humano ha evolucionado para ser activo por naturaleza, intrínsecamente motivado y orientado a desarrollarse de manera natural a través de diversos procesos integrativos. Estas cualidades son innatas en el ser humano, se desarrollan en el tiempo y se ven afectadas por el entorno social (Deci \& Ryan, 2012a).

Las investigaciones sobre los efectos de las recompensas extrínsecas en la motivación intrínseca dieron paso a la evolución de la Teoría de la Auto-Determinación. En los primeros estudios publicados por Deci (1971), los estudiantes de una universidad recibieron un pago monetario para que armen rompecabezas que eran intrínsecamente interesantes para ellos. Encontraron que la recompensa monetaria debilitó la motivación intrínseca que tenían los estudiantes por la actividad. Esta hipótesis fue comprobada en más de 128 estudios similares (Deci, Koestner, \& Ryan, 1999), donde se confirma que las recompensas no siempre motivan la persistencia en una actividad e incluso pueden debilitar el grado de la motivación intrínseca (Deci \& Ryan, 2012a).

La Teoría de la Auto-Determinación se ha desarrollado en forma de mini-Teorías, en la que cada una relata y explica un fenómeno específico. Las mini-Teorías están enlazadas en la medida que comparten supuestos organísmicos y dialécticos, y todos involucran el concepto 
de las necesidades psicológicas básicas. Cuando son coordinadas, las mini-Teorías, cubren todos los tipos de conducta humana en todos los ámbitos. Así, juntas constituyen la Teoría de la Auto-Determinación (Ryan \& Deci, 2002).

A continuación, se presentan brevemente la descripción de las cinco mini-teorías.

\section{Teoría de evaluación cognitiva}

La Teoría de la evaluación cognitiva fue formulada para describir los efectos del entorno social en la motivación intrínseca de las personas (Deci, 1975; Deci \& Ryan, 1980). Describe y categoriza los elementos en: apoyo a la autonomía, controladores o desmotivadores, y los enlaza con los diferentes tipos de motivación, sea intrínseca o extrínseca (Ryan \& Deci, 2002). Esta teoría se centra básicamente en el concepto de la motivación intrínseca. Una actividad que ha sido motivada intrínsecamente se realiza porque el comportamiento es interesante y genera una satisfacción de manera espontánea. La motivación intrínseca está vinculada con la tendencia innata de los seres humanos de explorar el mundo, desarrollar sus habilidades y conquistar desafíos. Las emociones asociadas con la motivación intrínseca son la alegría, el interés y la emoción. Muchas investigaciones sobre la evaluación cognitiva (Deci et al. 1999) han demostrado que la motivación intrínseca puede ser debilitada por recompensas extrínsecas, especialmente si estas contingencias externas son percibidas como controladoras, siendo algunas las recompensas monetarias o el sentido de poder o estatus social (Güntert, 2015).

\section{Teoría de la integración organísmica}

Los autores Deci y Ryan (1985b); Ryan y Connell (1989) refieren que la teoría se preocupa por la internalización e integración de los valores y normas. La integración 
organísmica fue formulada para explicar el desarrollo y las dinámicas de la motivación extrínseca; el grado en que los individuos experimentan autonomía mientras se involucran en actividades que han sido motivadas extrínsecamente; y los procesos mediante los cuales las personas adoptan los valores y costumbres de sus grupos y culturas (Ryan \& Deci, 2000).

Algunos estudios empíricos (Koestner, Ryan, Bernieri \& Holt, 1984; Ryan, 1982), postulan que, dentro del proceso de internalización, la motivación extrínseca puede variar según los grados en los que se experimenta como autónoma vs. controlada, por ello se distinguen diferentes tipos de motivación extrínseca (Ryan \& Connell, 1989).

A continuación, se detallan los cuatro tipos de motivación extrínseca. Se inicia la explicación por el tipo que refiere al menor grado de autonomía, se encuentra la regulación externa, donde la motivación de las personas proviene de obtener una recompensa o evitar un castigo. El siguiente tipo, es la regulación introyectada, donde las personas se sienten motivadas por la sensación de orgullo y autoestima, o para evitar sentimientos de vergüenza y culpa. El tercer tipo es la regulación identificada, donde las personas comprenden y aprueban el valor personal y significado de un comportamiento y como resultado experimentan un sentido de libertad al hacerlo. Finalmente, para lograr un proceso completo de internalización se debe asimilar las identificaciones previamente mencionadas y alinearlas con otros aspectos de uno mismo, conociéndose a este tipo como la regulación integrada. Esta última regulación consiste en la síntesis de las identificaciones mencionadas y a través de un proceso que requiere reflexión y auto-conciencia, se busca poder alcanzar un sentido unificado y coherente de uno mismo, que las acciones vayan acorde a los pensamientos y deseos (Vansteenkiste et al., 2010).

\section{Teoría de orientaciones causales}

Según Deci y Ryan (1985a), las orientaciones causales se formularon para describir las diferencias individuales que tienen las personas para dirigirse hacia el entorno social en formas 
que apoyan su propia autonomía, controlan su comportamiento o son desmotivadoras. Esta teoría permite predecir la orientación y las conductas a partir de las permanentes experiencias que tienen las personas (Ryan \& Deci, 2002).

Existen tres tipos de orientaciones causales. El primero, donde las personas con una alta orientación a la autonomía tienden a comportarse de acuerdo a sus propios intereses y valores, interpretan los eventos externos de manera informativa y regulan su comportamiento con autonomía. Por otro lado, aquellos que tienen una alta orientación hacia el control, tienden a comportarse de acuerdo a demandas internas o externas, interpretan los eventos como demandantes y regulan su conducta a través de acciones controladoras, como fechas límite, castigos, ultimátum, entre otros; este sería el segundo tipo de orientación. Finalmente, el tercer tipo es aquella donde las personas con una alta orientación impersonal perciben las experiencias de la vida como alejadas de su control personal, como consecuencia, son propensos a sentimientos invasivos de ineficiencia, impotencia e inactividad (Vansteenkiste et al., 2010).

\section{Teoría de las necesidades psicológicas básicas}

Según Ryan y Deci (2000), esta teoría fue formulada para explicar el concepto de necesidades psicológicas básicas y su relación con el bienestar físico y psicológico de las personas. Esta teoría postula que la satisfacción de las tres necesidades psicológicas básicas: autonomía, competencia y relación, predicen el funcionamiento óptimo y bienestar de las personas. Es por eso que los contextos que satisfacen o frustran estas necesidades tienen un impacto positivo o negativo respectivamente, en el bienestar general de los seres humanos (Ryan \& Deci, 2002).

La teoría propone que las tres necesidades psicológicas básicas son esenciales para mantener la motivación intrínseca, internalizar la motivación extrínseca e integrar la regulación 
de las emociones, todas las cuales están asociadas con el funcionamiento efectivo y el bienestar del ser humano (Deci, Ryan \& Guay 2013). Las tres necesidades psicológicas básicas serán descritas con mayor detalle más adelante.

De acuerdo a la Teoría de la Auto-Determinación, los contextos sociales tienen un impacto en los individuos, facilitando vs. perjudicando la satisfacción de las necesidades psicológicas básicas. Se define la palabra necesidad como los requisitos organísmicos para la salud. Las necesidades psicológicas son un subconjunto de estos requisitos organísmicos, las cuales son esenciales para el crecimiento psicológico, la integridad y bienestar. En la medida que estas necesidades sean satisfechas, las personas se desarrollarán de manera próspera y saludable, pero mientras estas necesidades se vean frustradas experimentarán daños psicológicos (Deci \& Ryan, 2012b).

\section{Teoría del contenido de las metas}

Según los estudios (Kasser \& Ryan, 1996), las metas en la vida o las aspiraciones intrínsecas incluyen el crecimiento personal, las relaciones cercanas, la contribución con la comunidad y la salud física; mientras que las metas extrínsecas incluyen la riqueza, la fama y la imagen. Las metas o aspiraciones intrínsecas son propensas a satisfacer las necesidades psicológicas básicas de autonomía, competencia y relación, mientras que las metas o aspiraciones extrínsecas son menos propensas a no tener relación alguna con la satisfacción de estas necesidades. Además, estudios señalaron que aquellas personas que tenían fuertes aspiraciones extrínsecas evidenciaron sentirse menos autorrealizados, con menor vitalidad y mayor depresión, ansiedad y narcisismo (Deci \& Ryan, 2012a). 


\section{Otras Teorías en el Estudio de la Motivación}

La presente investigación, estudia dos variables que han sido desarrolladas en el marco de la Teoría de la Auto-Determinación: Las necesidades psicológicas básicas y los dos estilos motivacionales, de apoyo a la autonomía o controlador. Sin embargo, es importante describir otras teorías de la motivación y resaltar las semejanzas o diferencias de éstas con la Teoría de la Auto-Determinación.

El psicólogo Fredrick Herzberg creó la Teoría de los dos factores de motivación, la cual propone que existe una relación entre los factores intrínsecos con la satisfacción laboral, y entre los factores extrínsecos y la insatisfacción. Dentro de los factores intrínsecos o motivadores se encuentra la realización, reconocimiento, responsabilidad, el progreso y el desarrollo de la persona. Los factores extrínsecos o higiénicos incluyen el salario, la administración, supervisión, relaciones interpersonales, políticas y estructura administrativa de la empresa. La diferencia entre dichos factores es que los factores extrínsecos o higiénicos son todos aquellos elementos externos al trabajo, no producen una motivación en sí, pero evitan la insatisfacción, funcionan como recompensas si existe un alto desempeño. Los factores intrínsecos son aquellos que sí producen motivación y corresponden al mundo interno de la persona, están vinculados directamente con la satisfacción laboral (Hezberg, 2003).

Una de las diferencias de la Teoría de Herzberg con la Teoría de la AutoDeterminación, es que ésta última considera que las relaciones interpersonales son un factor importante en la motivación intrínseca de la persona, y la define como una necesidad psicológica básica, bajo el nombre de relación, la cual debe ser satisfecha para que la persona pueda sentirse realizada. Mientras que la teoría de Herzberg propone que las relaciones interpersonales son un factor extrínseco.

La Teoría de Jerarquía ERC (Existencia, relación y crecimiento), creada por Clayton Alderfer, reduce las cinco necesidades propuestas por Abraham Maslow a tres. Alderfer 
propuso las siguientes: necesidad de existencia, se ocupa de satisfacer nuestros requerimientos básicos de bienestar fisiológico; necesidad de relación, el ser humano busca mantener relaciones interpersonales importantes y la necesidad de crecimiento, es el deseo intrínseco de desarrollo personal. Alderfer refiere que la satisfacción de las necesidades no tiene que ser sólo en movimiento ascendente de la jerarquía, las personas pueden retroceder en esta jerarquía en busca de satisfacer una necesidad que ha sido previamente satisfecha. La Teoría de Alderfer toma en consideración las diferencias individuales entre las personas. Aspectos como la educación, los antecedentes familiares y el ambiente cultural pueden determinar la importancia de satisfacer una necesidad antes que la otra (Alderfer, 1989).

De la misma manera que la Teoría de la Auto-Determinación, Alderfer propone tres necesidades para alcanzar la motivación. Ambas teorías tienen en común la necesidad de relación. Sin embargo la necesidad de crecimiento propuesto por Alderfer, vendría a ser el objetivo central en la Teoría de Auto-Determinación, donde el ser humano busca su desarrollo y crecimiento a través de la satisfacción de las tres necesidades psicológicas básicas.

Por último, la teoría de los tres factores de McClelland postula que la motivación laboral está en función de tres necesidades aprendidas y adquiridas a lo largo de la vida como resultado de las experiencias de cada persona. McClelland ve estas necesidades ve como cuestiones de personalidad, están latentes en el ser humano. Las tres necesidades propuestas son las siguientes: necesidad de logro, es el afán de alcanzar el éxito, realizarse y evitar el fracaso; de poder, es la necesidad de influir sobre los demás y ejercer control sobre ellos; y de afiliación, necesidad que busca tener relaciones interpersonales afectivas. Según McClelland, todas las personas tienen estas necesidades en mayor o menor grado, independientemente del género, edad o cultura. También, las personas pueden tener satisfechas las necesidades en diferentes grados, pero pueden haber sido motivadas de forma distinta (Uduji \& Ankeli, 2013). 
Nuevamente, la necesidad de afiliación es considerada dentro de las tres necesidades para lograr una motivación laboral. Esta necesidad es compartida entre tres teorías: AutoDeterminación, Teoría de ERC de Alderfer y Teoría de tres factores de McClelland, aunque cada una con definiciones distintas. Así, para la teoría de dos factores de Alderfer, la necesidad de relacionarse con los demás es considerado parte de la motivación extrínseca. En la teoría de necesidades sociales de McClelland al igual que la Teoría de la Auto-Determinación, postulan que las necesidades son universales, todas las personas tienen presente de manera innata y latente esas tres necesidades, indistintamente del genero, edad o cultura, pero pueden estar satisfechas en diferentes grados.

\section{Investigaciones sobre la motivación en Lima Metropolitana}

En el Perú, se han realizado estudios sobre la motivación empresarial. Se han evaluado las variables de productividad, liderazgo, motivación y rendimiento, entre otros. Los resultados de las presentes investigaciones que se verán a continuación son de utilidad para poder conocer cuál es la demanda de la motivación dentro del rubro empresarial de Lima Metropolitana y cómo afecta el desempeño de los trabajadores.

Una investigación realizada en Lima, Perú tomó como muestra a los trabajadores de la Gerencia de Infraestructura de una empresa de telefonía para evaluar el impacto de la motivación en la productividad empresarial. Se encontró que el factor de motivación influye en el desempeño de los trabajadores y es fundamental para alcanzar los objetivos propuestos. También se encontró que el carácter y actitud de los jefes ejerce una influencia en los colaboradores (Huamaní \& Acuña, 2015).

Gherman, Iturbe y Osorio (2011) realizaron un caso de estudio en base a la teoría de los dos factores de la motivación de Hertzberg, tomando como muestra a los trabajadores de una empresa del rubro Retail de Lima Metropolitana. Los resultados mostraron que los tres 
factores más importantes para la motivación fueron las relaciones interpersonales con los superiores y jefes, las relaciones interpersonales con los colegas y el factor de recomendación por parte del jefe o superior. Se puede concluir con los resultados de este estudio que el factor más importante para la motivación son las relaciones interpersonales, lo cual guarda una estrecha relación con la necesidad de relación que propone la Teoría de la Auto-Determinación.

Por último, una investigación realizada en una empresa de servicios de Lima Metropolitana tomó como muestra a 167 trabajadores para comprobar si la motivación y el liderazgo se relacionan con el rendimiento laboral. En los resultados se encontró que existe una correlación positiva y significativa entre la motivación y el liderazgo con la variable de rendimiento laboral. Mientras incrementa la motivación aumenta el rendimiento laboral y asimismo, cuando existe un mayor liderazgo por parte de los superiores el rendimiento laboral también es mayor. Este estudio comprueba que la motivación afecta directamente el desempeño y rendimiento laboral de los trabajadores (Huamaní \& Vargas, 2013).

De acuerdo a las investigaciones y estudios mencionados, se puede concluir que las relaciones interpersonales, sea con superiores o con colegas, cumple un papel importante para lograr una mayor motivación en los colaborares. A su vez la motivación y el estilo de liderazgo que utiliza el superior influye en la calidad del rendimiento laboral de los trabajadores. El factor de relaciones interpersonales guarda relación con la necesidad psicológica básica de relación propuesta por la Teoría de Auto-determinación, y el estilo de liderazgo con un estilo de motivación que apoya la autonomía, si se busca motivar a los trabajadores.

A continuación, se procederá a explicar las variables a estudiar en la presente investigación: las Necesidades Psicológicas Básicas. 


\section{Necesidades Psicológicas Básicas}

Ryan y Deci (2002) refieren que el concepto de necesidades ha recibido menos atención y aceptación en cuanto a propiedades psicológicas esenciales que las fisiológicas. La Teoría de la Auto-Determinación mantiene que existen condiciones necesarias para el crecimiento y bienestar de la personalidad de los seres humanos y sus estructuras cognitivas, así como son necesarias para su funcionamiento y desarrollo físico. Estas propiedades se denominan como necesidades psicológicas básicas.

Para calificar como necesidad, debe existir una relación directa entre la motivación y el bienestar. Cuando las necesidades son satisfechas, promueven bienestar, pero al ser obstaculizadas, desencadenan en consecuencias negativas. Bajo la Teoría de la AutoDeterminación, estas necesidades psicológicas básicas son universales, es decir, representan requerimientos innatos y no motivos adquiridos. Al ser necesidades universales, la relación entre la satisfacción y bienestar se aplica en todas las edades, géneros y culturas (Ryan \& Deci, 2002).

Las tres necesidades psicológicas básicas son la autonomía, la competencia y la necesidad de relacionarse con los demás. Dado que estas necesidades son esenciales para lograr una óptima salud psicológica, las personas tienden dirigirse hacia aquellas situaciones que promueven la satisfacción y se alejan de aquellas situaciones que evitan atender estas necesidades. En muchos casos, las personas no buscan satisfacer las necesidades de manera directa; sino, ellas realizan lo que les parece interesante y personalmente importante, y de ese modo, experimentan la satisfacción de las necesidades psicológicas básicas (Deci \& Vansteenkiste, 2004).

A continuación, se explican las necesidades una a una empezando por la autonomía. 


\section{Autonomía}

Deci y Ryan (2012b) definen la autonomía como la necesidad de poder actuar con un sentido de elección, disposición y voluntad propia. La satisfacción de la autonomía genera que las personas sientan que son los autores de sus propias acciones y actúen de forma coherente con sus intereses y valores. Esta necesidad está ligada al "yo", que es el centro de la integración, iniciación y compromisos de manera espontánea dentro del contexto social (Deci et al., 2013). Sin embargo, la frustración de esta necesidad involucra que la persona se sienta controlada a través de presiones externas, que son forzadas o impuestas por sí mismos (Chen et al., 2015; deCharms, 1968; Deci \& Ryan, 1985a).

Tener autonomía no significa ser independiente de otros, mas bien significa tener un sentido de voluntad y elección al momento de actuar, ya sea si las acciones son iniciadas independientemente por uno mismo o son una respuesta a una petición de otros (Chirkov, Ryan, Kim \& Kaplan, 2003; Deci \& Vansteenkiste, 2004).

\section{Competencia}

La necesidad de competencia está definida como el deseo en las personas de sentirse efectivos al interactuar con el ambiente y sentirse capaces de obtener los resultados deseados. Esta necesidad es importante en la medida que las personas puedan explorar y operar en su entorno; y comprometerse en tareas retadoras para poner a prueba y desarrollar sus habilidades. La satisfacción de la competencia permite a las personas adaptarse en ambientes complejos y cambiantes, mientras que la frustración de esta necesidad conlleva a la falta de motivación, sentimientos de fracaso y dudas sobre la eficacia de uno mismo (Deci \& Ryan, 2000; Chen et al., 2015; Van den Broeck, Vansteenkiste, De Witte, Soenens, \& Lens, 2010; White, 1959).

Esta necesidad conduce a las personas a buscar desafíos que se encuentren ligeramente fuera de sus capacidades actuales y a practicar diferentes tipos de actividades para 
eventualmente, mejorar sus aptitudes. Sin embargo, el nivel de aptitud que un individuo adquiere no es lo importante, sino más bien, lo importante es la experiencia de percibirse a uno mismo como competente (Deci et al., 2013).

\section{Relación}

La necesidad de relación está definida como el deseo de sentir una conexión con las demás personas alrededor. Esta conexión se da al ser miembro de un grupo, amar y cuidar de otros, y cuando la relación que se entabla es bidireccional, es decir, recibir de la otra persona la misma respuesta de deseo de conexión (Baumeister \& Leary, 1995). Esta necesidad es satisfecha cuando las personas experimentan un sentido de comunión y desarrollan relaciones íntimas y cercanas con otros (Deci \& Ryan, 2000). Por otro lado, la frustración de la necesidad de relación involucra sentimientos de exclusión y soledad (Chen et al., 2015; Van den Broeck et al., 2010).

La necesidad de relación refleja la tendencia de querer conectar con los demás, poder interactuar y ser aceptado por otros. La necesidad de sentir que uno mismo está relacionándose con los demás no por obtener un resultado determinado (ej: sexo, cierto status formal o convertirse en cónyuge o miembro de un grupo), sino se refiere al sentido psicológico de estar con otros en comunión o unidad (Ryan \& Deci, 2002).

A continuación, se procederá a explicar la siguiente variable a estudiar en esta investigación: los Estilos de Motivación. 


\section{Estilos de Motivación}

La teoría propone que los contextos sociales y continuas diferencias individuales ejercen una influencia en la satisfacción de las necesidades psicológicas básicas de las personas y por lo tanto también influyen en su performance, motivación y adaptación (Baard, Deci, \& Ryan, 2004). Como refiere la teoría, el estilo de motivación puede adoptarlo un individio como un contexto social. En esta investigación, se trabajó con el estilo de motivación que utiliza un individuo, en este caso un supersvisor. A continuación, se describirán los dos estilos de motivación que se trabajan.

Estilo de motivación de apoyo a la autonomía

Deci et al. (2001) y Williams, Gagne, Ryan y Deci (2002) refieren que un contexto o persona que apoya la autonomía se caracteriza por brindar la capacidad de elegir, fomentar la iniciativa, reconocer los sentimientos desarrollados hacia la actividad, transmitir confianza en las capacidades y fortalecer las habilidades en un ambiente de relación con los demás. De esta manera, se promueve una motivación autónoma (motivación intrínseca, identificada e integrada), a diferencia de una motivación de control (motivación extrínseca, introyectada y externa). Específicamente, la calidad de la interacción con personas que tienen una posición de autoridad, como son los profesores, gerentes, jefes y/o padres, puede afectar el grado de satisfacción de las necesidades psicológicas básicas de la persona y esto a su vez afecta el grado en que él o ella valora y disfruta de un objetivo o actividad (Gagne, 2003).

Dentro del entorno del trabajo, el apoyo a la autonomía se refiere a la orientación interpersonal del propio gerente o supervisor con su subordinado al reconocerlo y lograr comprender la perspectiva de éste. Además, el supervisor que tiene un estilo de apoyo a la autonomía proporciona oportunidades para que los empleados tengan libertad sobre lo que hacen y cómo lo hacen, fomentan la iniciativa, permanecen abiertos a nuevas experiencias y 
desarrollan los recursos internos de motivación que tenga el empleado (Baard et al., 2004; Deci \& Ryan, 1987; Moreau \& Mageau, 2012; Slemp, Kern, \& Vella-Brodrick, 2015).

Algunos estudios teóricos (Deci et al., 1989; Deci, Eghrari, Patrick, \& Leone, 1994; Richer \& Vallerand, 1995), han identificado la importancia de los siguientes cuatro comportamientos específicos que pueden tener los gerentes para apoyar la autonomía de sus empleados: promover los recursos motivacionales internos (conocer los intereses y preferencias de cada empleado y encontrar funciones donde se pongan en práctica), evitar un lenguaje controlador (no dar mensajes rígidos, evaluadores y que produzcan presión), brindar explicaciones a los requerimientos solicitados (siempre explicar el por qué de una solicitud y su utilidad en el negocio). Por ultimo, reconocer y aceptar las expresiones de afecto negativo de los empleados (escuchar y aceptar las quejas y desacuerdos) (Hardré \& Reeve, 2009).

\section{Estilo de motivación de control}

Según Reeve (2009) una autoridad con un estilo de motivación controlador presiona a otros a pensar, sentir o comportarse de una manera específica, busca influenciar en sus pensamientos y fuerza a las personas a que adopten su misma perspectiva, ignorando sus sentimientos y necesidades personales. Un estilo de motivación de control opera a través de conductas seductoras que incluyen recompensas explícitas o implícitas, o castigos. Otras conductas incluyen: la negación de la racionalidad, utilizar un lenguaje que induce presión, mostrar impaciencia por ver las cosas bien hechas y afirmar su poder para superar las quejas y las expresiones negativas de los otros (Amoura, Berjot, Gillet, Caruana, \& Finez, 2015; Black \& Deci, 2000).

En relación con el tipo de motivación, las investigaciones han demostrado que un estilo motivacional de control, se relaciona con la motivación controlada (regulación introyectada y externa) y con la falta de motivación de los subordinados. En otras palabras, cuando un 
supervisor utiliza un estilo de motivación de control, las necesidades psicológicas básicas de los subordinados se ven frustradas y la motivación es menos auto-determinada (Amoura et al., 2015; Pelletier, Fortier, Vallerand, \& Brière, 2001; Soenens, Sierens, Vansteenkiste, Dochy, \& Goossens, 2012).

En cuanto al ámbito organizacional, generalmente cuando el supervisor da una orden, la motivación interna de sus empleados se ve obstaculizada y éstos se sienten presionados a comportarse de una manera específica (Deci \& Ryan, 1985b; Reeve, 2009). Los supervisores con un estilo motivacional controlador utilizan incentivos extrínsecos, indicaciones, tareas con tiempos límite, evaluaciones y requerimientos complicados que están separados o relacionados de manera arbitraria a la actividad. Por ejemplo, ofrecerle a un empleado un bono o incentivo si él o ella aumenta las ventas (Hardré \& Reeve, 2009).

\section{Investigaciones sobre el estilo de motivación y la satisfacción de las necesidades psicológicas básicas}

Se han realizado investigaciones en diferentes contextos sobre el estilo de motivación que utilizan las personas que tienen personal a su cargo y cómo el estilo motivacional es percibido por el personal a cargo. Los contextos de estas investigaciones son: empresarial, educacional, deportivo, familiar y de salud. Los instrumentos utilizados en las investigaciones que tienen como objetivo determinar el estilo de motivación de un individuo con personal a cargo son: Cuestionario de Problemas en el Trabajo (PAW) y el Cuestionario de Problemas en la Escuela (PIS), entre otros. En otros estudios se evaluó el estilo motivacional que el subordinado pudo percibir de su supervisor, se utilizaron los siguientes instrumentos: Escala del Apoyo de la Autonomía Percibida sobre los Padres, Cuestionario sobre la Percepción de Apoyo a la Autonomía, y Cuestionario del Apoyo a la Autonomía Percibida sobre el coach deportivo, entre otros. 
A continuación, se describirán las investigaciones realizadas en el contexto empresarial sobre la relación que existe entre el estilo de motivación utilizado por los supervisores, especialmente el de apoyo a la autonomía, y la satisfacción de las necesidades psicológicas básicas de sus empleados.

En una investigación realizada por Baard et al. (2004), se analizaron las siguientes variables en una muestra de 59 empleados: el nivel de autonomía de los empleados, la percepción del apoyo a la autonomía que tenían los empleados de sus supervisores, la satisfacción de las tres necesidades psicológicas básicas, el performance en el trabajo y el grado de ansiedad de los empleados. Los resultados de esta investigación señalan que el performance del empleado y la adaptación psicológica se relacionan con el grado en que sus necesidades psicológicas básicas (competencia, autonomía, relación) fueron satisfechas en el trabajo. También se encontró que mientras fuera mayor la percepción de un estilo motivacional favorable sobre un gerente, la satisfacción de las necesidades psicológicas básicas reportada por los trabajadores era mayor.

En un siguiente estudio, Gillet et al. (2012a) examinaron una muestra de 468 empleados para analizar la relación entre el apoyo organizacional percibido, la percepción del estilo de motivacional del supervisor, la satisfacción de las necesidades psicológicas básicas y el bienestar en los empleados. En los resultados se encontró que cuando los empleados percibían un estilo de apoyo a la autonomía en sus supervisores, también se reportó una mayor satisfacción de sus necesidades psicológicas básicas. En otras palabras, mientras los empleados percibían mayor apoyo a la autonomía del supervisor, sus necesidades de autonomía, competencia y relación eran satisfechas en mayor grado. También se encontró que un estilo de motivación controlador por parte del supervisor se asociaba negativamente con la satisfacción de las necesidades psicológicas básicas en los empleados. Es decir, cuando un supervisor se comporta de manera autoritaria e impone una forma específica de pensar y actuar 
sobre sus empleados, las personas no sienten que pueden satisfacer sus necesidades de autonomía, competencia y relación.

Muchas de las investigaciones sobre la Teoría Auto-Determinación en ambientes laborales se han realizado en un país con énfasis en el individualismo como lo es Estados Unidos (Deci et al., 2001). Por eso, es importante revisar un estudio comparativo de culturas realizado por Deci et al. (2001) en dos culturas diferentes, Estados Unidos y Bulgaria que tiene una cultura más bien colectivista. El estudio reveló que la satisfacción de las tres necesidades psicológicas básicas promueve la motivación y el bienestar en las culturas estudiadas, lo cual permite señalar que independientemente de una cultura individualista o colectivista, se encuentra el mismo tipo de relación entre el estilo motivacional de apoyo a la autonomía y la satisfacción de necesidades psicológicas básicas. El grado de apoyo a la autonomía en el ambiente de trabajo sí predijo la satisfacción de las necesidades básicas en cada cultura, y la satisfacción de estas necesidades además predijo el compromiso con la tarea y el bienestar. Por ello, este estudio brinda evidencia de la universalidad de las necesidades psicológicas básicas. Sin embargo, continuando con los resultados de Deci et al. (2001), algunas diferencias entre las culturas, es que los trabajadores en Bulgaria reportaron experimentar una gran satisfacción de la necesidad de autonomía, a diferencia de los trabajadores estadounidenses. Además, los trabajadores en Bulgaria reportaron tener un gran apoyo a la autonomía de sus supervisores inmediatos en el ambiente de trabajo, más que los estadounidenses. 


\section{Justificación y Pregunta de Investigación}

De acuerdo a lo expuesto anteriormente, resuelta interesante estudiar el nivel de satisfacción de las necesidades psicológicas básicas y cómo éstas pueden predecir un estilo motivacional favorable, que es el de apoyo a la autonomía. Dentro del ámbito organizacional, un estudio analizó la satisfacción de las necesidades psicológicas básicas y la motivación autónoma, y los resultados indicaron que aquellos empleados que tuvieron un mayor nivel de satisfacción de las necesidades psicológicas básicas en el trabajo, también mostraron un gran nivel de motivación autónoma. Es así como una motivación intrínseca y basada en la autonomía depende de la satisfacción de las necesidades básicas (De Cooman, Stynen, Van den Broeck, Sels \& De Witte, 2013).

Además, aquellos ambientes de trabajo que apoyan la satisfacción de estas necesidades no sólo facilitan la motivación autónoma de los empleados, sino también favorecen el bienestar físico y psicológico de los empleados, y su performance en el trabajo (Deci, Olafsen \& Ryan, 2017). Es así, como la satisfacción de las tres necesidades psicológicas básicas pueden facilitar una motivación favorable, el cual a su vez genera beneficios para los trabajadores de las empresas.

Así mismo, resulta importante identificar los estilos motivacionales y qué los predice, ya que las ventajas y beneficios que ofrece un estilo de motivación favorable generan un impacto positivo en el empleado y en la empresa. Arshadi (2010) realizó un estudio con empleados de una compañía del sector industrial en Irán. La data del estudio mostró que el apoyo a la a la autonomía de los jefes está relacionado con la satisfacción de las necesidades psicológicas básicas de los empleados a cargo, así como con su motivación y su performance en el trabajo. Por ello, cuando los gerentes tienen en consideración las necesidades de sus subordinados, deben de brindarles las condiciones de trabajo que faciliten la satisfacción de estas necesidades y que conlleven a resultados positivos en el trabajo. 
Como se ha mencionado anteriormente, la práctica de los estilos motivacionales tienen ventajas y desventajas. Hon (2011) realizó una investigación con los empleados de un hotel, y encontró que cuando los gerentes apoyaban la autonomía y los compañeros se relacionaban con los demás, los empleados estaban motivados a través de la autonomía y podían ser más creativos en su trabajo. Pero si los gerentes adoptaban un estilo motivacional controlador ejerciendo presión, los empleados se sentían desmotivados y menos creativos (Deci et al., 2017).

Es por eso que los resultados que se obtengan serán útiles para el sector empresarial, en especial aquellos trabajadores que tienen personal bajo su cargo, ya que podrán conocer sobre los dos estilos que existen para motivar a las personas, y las ventajas que existen al utilizar un estilo favorable de la motivación como lo es la autonomía y las desventajas de un estilo motivacional controlador. Esta investigación será compartida con el público de manera digital, de esta manera tendrá un mayor alcance para todos aquellos que busquen información sobre los estilos de motivación y la satisfacción de las necesidades psicológicas básicas en las empresas de Lima Metropolitana.

En cuanto al contexto del estudio de la presente investigación, dentro del Perú, se han realizado investigaciones sobre la percepción del estilo motivacional en el sector educacional. Spencer (2017) realizó un un estudio sobre el estilo motivacional docente, tipos de motivación, autoeficacia, compromiso agente y rendimiento en matemáticas. Se tomó una muestra de 165 estudiantes de dos universidades públicas. Los resultados evidenciaron que el estilo motivacional del docente de apoyo a la autonomía y de control, predicen respectivamente la motivación autónoma y controlada en los estudiantes.

Existen investigaciones sobre el estilo motivacional y la satisfacción de necesidades psicológicas básicas en el Perú. Sin embargo, estas investigaciones han sido realizadas en el contexto educativo. Por ello, el contexto de estudio de la presente investigación es relevante, 
ya que se estudiará la satisfacción de necesidades psicológicas básicas y los estilos de motivación en el ámbito laboral. Además, los instrumentos se aplicarán sólo en los supervisores, logrando identificar el estilo motivacional que ellos mismos utilizan y no el que perciben sus subordinados.

Es por eso, que el problema de esta investigación se desarrolló a partir de la pregunta de: ¿Cómo se relacionan el nivel de satisfacción de las necesidades psicológicas básicas y los estilos de motivación de los supervisores dentro del ámbito laboral en empresas de Lima Metropolitana?

En cuanto a la metodología, uno de los instrumentos utilizados en esta investigación es el Cuestionario de Problemas en el Trabajo (PAW), el cual solo existía en el idioma inglés. Se realizó una traducción formal al español, la cual fue evaluada por tres jurados. Es por eso que la presente investigación tiene una utilidad metodológica, ya que se desarrolló una versión al español del Cuestionario de Problemas en el trabajo (PAW), el cual puede ser utilizado para posteriores investigaciones. Y, por otro lado, se usó la Escala de Satisfacción y Frustración de Necesidades Psicológicas Básicas (BPNSFS) utilizada por Chen et al. (2015) en la cual se trabajó con una versión al español aplicada a un grupo de estudiantes universitarios peruanos. El aporte es que, en esta tesis, se ha aplicado en una muestra de supervisores en el ámbito organizacional, donde antes no había sido aplicado, obteniendo una validez y confiabilidad de la muestra.

Por último, los resultados de esta investigación serán de utilidad para investigadores que deseen realizar estudios sobre la motivación en las empresas, podrán conocer y utilizar los resultados que se obtengan acerca de la relación entre el grado de satisfacción de necesidades y estilo de motivación de los supervisores. También a partir de esta investigación podrán surgir ideas, recomendaciones y limitaciones para estudios futuros sobre el estilo de motivación y las necesidades psicológicas básicas en el rubro empresarial. 


\section{Capítulo 2: Método}

\section{Diseño y Tipo de investigación}

Esta investigación corresponde a un diseño correlacional cuantitativo (Hernández, Fernández \& Baptista, 2010), ya que se busca medir y estudiar la relación entre dos variables: las necesidades psicológicas básicas del supervisor y el estilo de motivación que utiliza. Además, es una investigación descriptiva y transversal porque se busca explicar y describir el grado de asociación de ambas variables en un determinado momento del tiempo.

\section{Participantes (población y muestra)}

La población de esta investigación son supervisores de empresas de Lima Metropolitana. El término supervisores incluye a gerentes, jefes y/o mandos medios, pero deben tener personal a su cargo. La muestra está compuesta por 111 supervisores de empresas de Lima Metropolitana. El muestreo fue no probabilístico intencional, ya que el número de participantes debe de reunir ciertas condiciones y una de ellas es que los supervisores deben tener personal a su cargo.

En cuanto a las características demográficas de la muestra el $62 \%$ fueron hombres y $38 \%$ mujeres, con una edad promedio de 38.6 años. La posición que desempeñaban en el momento estaba distribuida de la siguiente manera: $36 \%$ gerentes, $31 \%$ jefes, $16 \%$ supervisores, $11 \%$ analistas, $4 \%$ socios y subgerentes el $2 \%$.

El requisito para llenar la prueba fue tener mínimo una persona a su cargo y en la muestra se encontraron que el 29\% tenía 3 a 5 personas a cargo, el $24 \% 1$ a 2 personas a cargo, el $18 \% 6$ a 10 personas a cargo, el $15 \% 11$ a 20 personas, el $11 \% 50$ a más y el $4 \% 21$ a 50 personas. 
Es importante considerar el tiempo que venían trabajando con su equipo de trabajo actual. El 35\% de los trabajadores venía laborando 1 año o menos con su actual equipo, el $33 \% 1$ a 3 años, el 18\% 3 a 6 años y el $15 \% 6$ años a más.

En cuanto al área de trabajo en la que laboraban, el 22\% pertenecía al área de recursos humanos, el $18 \%$ al área de comercial y ventas, el 15\% en el área de contabilidad, el 13\% en sistemas, el $9 \%$ en finanzas y el $27 \%$ repartido en otras áreas de las empresas.

El rubro de las empresas de donde provenía la muestra fue el siguiente: el $44 \%$ del rubro de servicios administrativos, el 15\% del rubro de farmacéutico, el 15\% de un outsourcing de contabilidad, el 9\% del rubro de minería y construcción, el $7 \%$ de consultoría, el $4 \%$ en el rubro de bienes de consumo masivo, $4 \%$ en el rubro de seguros y $2 \%$ en el rubro consultoría legal.

\section{Instrumentos}

\section{Escala de satisfacción y frustacción de las Necesidades Psicológicas Básicas (BPNSFS)}

Para medir la variable de necesidades psicológicas básicas de los supervisores en empresas de Lima Metropolitana, utilizaremos el instrumento de "Basic Psychological Needs Satisfaction and Frustration Scale" o la Escala de Satisfacción y frustración de las Necesidades Psicológicas Básicas (Chen et al., 2015).

Esta escala mide la satisfacción de las necesidades psicológicas básicas y la frustración de estas. Sin embargo, en esta investigación se trabajará con los ítems que miden la satisfacción.

La escala está compuesta por 24 ítems, que miden la satisfacción y frustración de las necesidades psicológicas básicas. Los ítems que miden la sub-escala de satisfacción de la 
autonomía son: 1, 4, 7 y 10; la satisfacción de la competencia: 3, 6, 9 y 12; la satisfacción de relación: 2, 5, 8 y 11; la sub-escala de frustración de la autonomía: 13, 16, 19 y 22; la frustración de relación: 14, 17, 20 y 23; y la frustración de la necesidad de competencia: 15, 18, 21 y 24.

Validez y confiabilidad

La validez y confiabilidad de la Escala de Satisfacción y Frustración de las Necesidades Psicológicas Básicas fue probada en Chen et al, (2015). En este estudio se aplicó el instrumento en una muestra de 1051 personas. La muestra proviene de 4 países diferentes: China (309 personas), Estados Unidos (298 personas), Bélgica (200 personas) y Perú (244 personas).

Como se puede ver (tabla 1) se comprueba, según Chen et al. (2015), la consistencia interna en la muestra de estudiantes universitarios peruanos para las sub-escalas de autonomía, competencia y relación fue de $.80, .80$ y .73 respectivamente.

Tabla 1: Confiabilidad en la Escala de Satisfacción y Frustración de Necesidades Psicológicas Básicas en cuatro países

\begin{tabular}{lcccc}
\hline & EEUU & CHINA & PERU & BELGICA \\
\hline Puntuación Compuesta & .82 & .77 & .80 & .85 \\
Autonomía & .87 & .79 & .73 & .83 \\
Relación & .89 & .79 & .80 & .88 \\
Competencia & .81 & .70 & .74 & .82 \\
Satisfacción & .83 & .72 & .75 & .83 \\
Autonomía & .88 & .73 & .78 & .82 \\
Relación & & & & \\
Competencia & & & & \\
Frustración & & & & \\
\end{tabular}




\begin{tabular}{lllll}
\hline Autonomía & .71 & .72 & .77 & .77 \\
Relación & .81 & .73 & .64 & .67 \\
Competencia & .86 & .76 & .67 & .84 \\
\hline
\end{tabular}

La validez del instrumento fue comprobada por los resultados obtenidos en un análisis de regresión de las variables de necesidades psicológicas básicas en relación a la variable de bienestar, donde se obtuvo lo siguiente: autonomía $(.42 * *)$, competencia $(.28 * *)$ y relación $(.34 * *)$, los valores son significativos y la necesidad psicológica básica que tuvo mayor relación con la variable de bienestar fue la de autonomía. Además, el modelo fue validado en los cuatro países obteniendo: el Chi-cuadrado de Satorra-Bentler SBS-X2 $=(231)=441.99$. El índice de ajuste comparativo CFI $=0.95$, el cual debe ser mayor o igual a .95 para indicar un buen ajuste. La raíz cuadrada media del error de aproximación, el RMSEA=0.04, un valor igual o menor a 0.6 es considerado buen ajuste. Por último, la raíz cuadrada media residual estandarizada, $\mathrm{SRMR}=0.04$, el cual debe ser igual o menor a 0.9. Se puede concluir que los resultados indican que modelo se ajusta bien a los datos.

En cuanto a la forma de calificación de los ítems de la Escala de Satisfacción y Frustración de Necesidades Psicológicas Básicas (BPNSFS), se realiza a través de una escala Likert que va del 1 (definitivamente no es verdadero) al 7 (definitivamente verdadero), donde el participante, en este caso el supervisor, califica con un puntaje del 1 al 7, en qué medida se siente identificado con lo que señala el ítem. Al tener el puntaje del ítem, se obtiene un puntaje de la sub-escala que mide dicho ítem.

En seguida, se presentará un ítem de cada sub-escala. Solo se trabajará con la subescala de satisfacción de las necesidades psicológicas básicas. Un ítem de la prueba que mide la satisfacción de autonomía sería: "Siento que tengo la libertad y la posibilidad de elegir las 
cosas que asumo", un ítem de competencia sería: "Siento que soy capaz de alcanzar mis metas". Por último, un ítem que mide la relación sería: "Siento que le importo a las personas que me importan”.

\section{Cuestionario de problemas en el trabajo (PAW).}

Para medir la variable de estilo de motivación del supervisor se utilizará el Cuestionario de Problemas en el trabajo, "The Problems at Work Questionnaire” (PAW). Este instrumento fue diseñado, creado y validado en un estudio por Deci et al. (1989).

El cuestionario de problemas en el trabajo ha sido utilizado en investigaciones como: Baard et al., 2004; Deci et al., 1989. Este cuestionario está diseñado para que los supervisores se califiquen a ellos mismos sobre cómo piensan que motivan a sus empleados. Sin embargo, en el estudio realizado por Baard et al. (2004), se efectuó una adaptación del instrumento para que los subordinados lo completen brindado información sobre la percepción que tienen de cómo los motiva su propio supervisor. Cabe resaltar que este instrumento no ha sido utilizado en Latino América, hasta donde se ha revisado en este momento.

Como ya se mencionó previamente en el marco teórico, existen dos estilos de motivación, motivación de apoyo al control y de apoyo a la autonomía. Se explicará brevemente los dos estilos de motivación que evalúa el Cuestionario de Problemas en el Trabajo (PAW).

El estilo de motivación de apoyo a la autonomía se da cuando el individuo que se encuentra en la posición de autoridad, toma la perspectiva, conocimientos y sentimientos de la otra persona de menor autoridad, y le brinda información valiosa, le da la oportunidad de elegir y evita realizar demandas, minimizando las presiones. Por ejemplo, un profesor que apoya la autonomía, le brinda a los estudiantes la información necesaria para desarrollar un problema y 
al mismo tiempo los alienta para que utilicen dicha información y resuelvan el problema por si mismos (Black \& Deci, 2000; Deci \& Ryan, 1985b).

El estilo de motivación de control se da cuando una autoridad ejerce presión en las otras personas, ya sea de manera forzosa o con técnicas seductoras, para que se comporten de cierta manera, las cuales generalmente vienen acompañadas de recompensas implícitas o explícitas, o castigos. Un ejemplo podría ser el un profesor que le dice a los alumnos que tienen que resolver los problemas de una manera en particular para que les pueda ir bien en el examen (Black \& Deci, 2000; Deci \& Ryan, 1985b).

A continuación, se detallará la composición del Cuestionario de Problemas en el Trabajo o "Problems at Work Questionnaire" (PAW). El cuestionario está formado por 8 viñetas, cada uno describe un problema típico que el supervisor puede encontrar con su subordinado. Cada viñeta cuenta con 4 ítems, los cuales representan las cuatro sub-escalas que miden el grado del estilo de motivación utilizados para lidiar con el problema que hace referencia la viñeta, teniendo un total de 32 ítems. En la presente investigación, anteriormente se describió los dos estilos de motivación que existen: De apoyo a la autonomía y de apoyo al control. Sin embargo, este cuestionario ha dividido cada estilo de motivación en dos categorías: alto y moderado, teniendo como resultado 4 sub-escalas en el instrumento. En las siguientes líneas se detallará brevemente las cuatro sub-escalas, el número de ítem que representa cada sub-escala y un ejemplo para cada una de ellas:

Alto apoyo a la Autonomía (AA). El número de los ítems que miden esta sub-escala son 2, 5, 11, 13, 18, 23, 28 y 29. Ejemplo de un ítem: “Tener discusiones con el grupo para facilitar la elaboración de estrategias de los miembros y mejorar los resultados”.

Moderado apoyo a la Autonomía (MA). El número de los ítems que miden esta sub-escala son 4, 7, 10, 15, 20, 21, 26 y 31. Ejemplo de un ítem: "Enseñarle a mi subordinado algunas maneras que utilizan los otros empleados para relacionarse con sus clientes, para que pueda comparar 
su propio estilo con el de ellos". Altamente Controlador (AC). El número de los ítems que miden esta sub-escala son 3, 8, 9, 16, 17, 22, 27 y 32. Ejemplo de un ítem: "Insistir en que las órdenes deben de llevarse a cabo en un específico límite de tiempo y chequear que él esté cumpliendo con esas fechas límite”. Moderadamente Controlador (MC). El número de los ítems que miden esta sub-escala son 1, 6, 12, 14, 19, 24, 25 y 30. Ejemplo de un ítem: "Inculcar en ella que debe seguir su horario de trabajo por su propio bien".

En seguida se explicará la forma de calificación por el participante de los ítems del cuestionario. Los participantes leerán las 8 viñetas que se presentan y califican cada uno de los cuatro ítems de cada viñeta, un total de 32 ítems. Esta calificación se realizará a través de una escala Likert que va del 1 (definitivamente no es verdadero) al 7 (definitivamente verdadero), donde el participante, en este caso el supervisor, indica con un puntaje del 1 al 7 , en qué medida se siente identificado con lo que refiere cada ítem.

Adaptación lingüística del cuestionario problemas en el trabajo (PAW).

Para la adaptación de la prueba se realizó una traducción del idioma original inglés al español y se calificó la traducción a partir de un criterio de jueces, los cuales eran todos psicólogos, laboraban en el ámbito organizacional y tenían dominio del idioma inglés. Una vez escogido el jurado, comprendido por 3 personas, se les entregó un sobre personalmente que contenía el instrumento para su evaluación.

Una vez entregados los cuestionarios por los jueces, se analizó el nivel de acuerdo que se tenía entre ellos. En el caso que este fuera del $80 \%$, se consideraría como efectiva la adaptación lingüística.

La traducción del cuestionario empezó con la sección de indicaciones, la cual obtuvo un $80 \%$ de aceptación por parte de los jueces. Por otro lado, se evaluó el nivel de aprobación obtenido en cuanto a los comentarios de los jueces para las viñetas y opciones de respuesta del 
cuestionario. Los ocho ítems (viñetas) que corresponden a las situaciones, ítems 2 y 6 , obtuvieron una aceptación de $33 \%$ mientras que los ítems 1, 3, 4, 5,7 y 8 obtuvieron una aceptación del 50\%. En relación a las opciones de la prueba, 8, 10 y 30, obtuvieron un 100\% de aceptación, las opciones 5, 6, 9, 11,15, 19, 21, 23, 24, 25, 29 y 31 obtuvieron una aceptación del $83.33 \%$. Las opciones $1,4,7,13,14,20$ y 26 una aceptación de $66.6 \%$, las opciones 3, 12, 16, 18, 22, 27, 28 y 32 una aceptación de 50\% y finalmente, las opciones 2 y 17 una aceptación de $50 \%$.

Dentro de la calificación se mantuvo para la traducción aquellos ítems que obtuvieron un porcentaje de aceptación mayor o igual a 80\%, luego se procedió a realizar un análisis de las sugerencias de los jueces para todos aquellos ítems que obtuvieron una puntuación menor a la mencionada y se modificaron los ítems según la sugerencia de los expertos. De esta manera se concluyó satisfactoria mente con la traducción de la prueba.

\section{Validez y confiabilidad.}

La validez y confiabilidad se estudió en una investigación realizada por Deci et al. (1989) donde los gerentes de una empresa completaron el Cuestionario de Problemas en el Trabajo (PAW) en tres diferentes momentos en un periodo de dos años. El Alfa de Cronbach fue mayor a .70 en la primera aplicación y mayor a .75 en la tercera aplicación, indicando una buena consistencia interna. La confiabilidad del test-retest fue de .80 , a lo largo de un periodo de cuatro meses, lo cual indica una buena estabilidad temporal. La evidencia de la validez fue dada por la correlación significativa del estilo de motivación de los gerentes con el puntaje de las de las siguientes variables de satisfacción de los subordinados: confianza .72, no sentir presión .61, calidad del feedback .57, oportunidad para inputs .71, seguridad .60, confianza en la organización .55, potencial para desarrollo .53 y satisfacción general .69. 


\section{Procedimiento de Recolección y Procesamiento de la Información}

En esta sección se explica cómo se realizó la administración de los instrumentos. Ambos instrumentos, la Escala de Satisfacción y Frustración de Necesidades Psicológicas Básicas (BPNSFS) y el Cuestionario de Problemas en el Trabajo (PAW), fueron aplicados a 111 supervisores que trabajan en empresas de Lima Metropolitana, los cuales pueden tener el cargo de jefes, gerentes, analistas etc. El único requisito fue que tuvieran personal a su cargo para supervisar.

Para cuidar los aspectos éticos de esta investigación se entregó a cada evaluado un consentimiento informado, y así se les comentó sobre el objetivo de la investigación así como también el carácter anónimo y confidencial de la información brindada. Asimismo se les señaló que su participación era totalmente voluntaria.

Como parte del procedimiento, se adjuntó una hoja de datos generales, la cual contenía preguntas para marcar sobre sus características personales y profesionales; por ejemplo, si era casado o soltero, o si ha recibido alguna capacitación en métodos de supervisión o retroalimentación, entre otras preguntas. Luego, se les brindó la consigna e instrucciones específicas para poder desarrollar cada instrumento.

Además, se seleccionó una muestra que cumplía con los siguientes requisitos: tener el puesto supervisor, contar con personal a su cargo y trabajar en una empresa de Lima Metropolitana. Una vez finalizada la aplicación, se recolectó los instrumentos, se revisó que las respuestas estén completas y se precedió a realizar la puntuación de cada sub-escala. Para finalizar, se utilizó el software estadístico SPSS "Statistical Package for the Social Sciences” para introducir la información y realizar el análisis estadístico de los resultados. Una vez obtenidos los resultados, se procedió con la discusión y las conclusiones. 


\section{Capítulo 3: Resultados}

\section{Propiedades Psicométricas de los Instrumentos utilizados: Validez y Confiabilidad}

Validez de la Escala de Satisfacción y Frustración de Necesidades Psicológicas Básicas (BPNSFS)

Evidencias de validez de constructo.

Debido a que se conoce la estructura factorial de la prueba se decidió estudiar las evidencias de validez de constructo a través de un análisis factorial confirmatorio (AFC). Así se pudo especificar de antemano la estructura de los factores utilizando como base la fundamentación empírica y conceptual de la Teoría de la Autodeterminación.

Los índices de ajuste del modelo fueron satisfactorios: $\mathrm{X} 2(\mathrm{gl}=51)=172.83$, RMSEA=.059, SRMR=.070, CFI=099. El RMSEA, es el error cuadrático medio de aproximación, y al ser $\leq .06$, se considera un ajuste aceptable de los datos (Hu \& Bentler, 1999). El SRMR es la raíz del residuo estandarizado medio, se obtuvo un valor inferior a .08, lo cual indica un excelente ajuste (Simms, Watson \& Doebbeling, 2002). Por último, el CFI es el índice de ajuste comparativo, se obtuvo un valor $\geq .95$, indicando la aceptación del modelo propuesto (Hu \& Bentler, 1999).

De esta manera los resultados obtenidos brindan buenas evidencias sobre la estructura factorial planteada en la muestra estudiada, lo cual confirma la validez de la prueba. 


\section{Confiabilidad de la Escala de Satisfacción y Frustración de Necesidades Psicológicas Básicas (BPNSFS)}

Para estudiar las evidencias de confiabilidad de la Escala de Satisfacción y Frustración de Necesidades Psicológicas Básicas (BPNSFS), se utilizó el método de consistencia interna utilizando el coeficiente Alfa de Cronbach. Este método permite medir la fiabilidad del instrumento y asume que los ítems miden un mismo constructo y que están altamente correlacionadas entre sí. Los valores van del 0 al 1, siendo el más cercano al uno, el más significativo (Cronbach, 1951). También, se utilizó la correlación elemento-total corregida para poder analizar la correlación lineal entre el ítem y la puntuación total y así señalar el grado y dirección de esta relación, las correlaciones a partir de .30 son estadísticamente significativas (Field, 2009).

En las tablas de las sub-escalas se presenta el valor de la media de la escala si se elimina el elemento, indica el valor que tendría la media, es decir el promedio de los ítems, en caso de eliminar cada uno de los elementos. Por último, el valor de la varianza de la escala si se elimina el elemento indica el grado de dispersión respecto a la media si se elimina uno de los elementos; en otras palabras, indica cuán alejados o cuán cercanos se encuentran los datos respecto a la media, a más dispersión el conjunto de los datos de la muestra es más heterogénea, y a menor dispersión, los datos son más homogéneos, indicando que la medida de tendencia central es más representativa de los datos. A continuación, se presenta el análisis de confiabilidad para cada sub-escala.

En la sub-escala de autonomía (Ver tabla 2) se obtuvo un coeficiente de consistencia interna Alfa de Cronbach de .687 el cual se considera aceptable y las correlaciones elementototal corregida se encontraron entre .440 y .502 , los cuales son mayores a .30 , siendo estadísticamente significativas (Field, 2009). 
Tabla 2: Autonomía: Correlación Elemento-Total Corregidas

Estadísticos total-elemento

\begin{tabular}{lcccc}
\hline & $\begin{array}{c}\text { Media de la escala si } \\
\text { se elimina el } \\
\text { elemento }\end{array}$ & $\begin{array}{c}\text { Varianza de la } \\
\text { escala si se elimina } \\
\text { el elemento }\end{array}$ & $\begin{array}{c}\text { Correlación } \\
\text { elemento-total }\end{array}$ & $\begin{array}{c}\text { Alfa de Cronbach si } \\
\text { se elimina el } \\
\text { corregida }\end{array}$ \\
\hline N1 & 17.63 & 4.878 &. $\mathbf{4 4 0}$ & .661 \\
N7 & 17.25 & 6.100 & .534 & .599 \\
N10 & 17.43 & 6.192 & .462 & .632 \\
\hline
\end{tabular}

En la sub-escala de Competencia (Ver tabla 3) se obtuvo un coeficiente de consistencia Alfa de Cronbach de .780 el cual es un buen valor y las correlaciones elemento total corregida se encontraron entre .620 y .523 , siendo mayores a .30 son estadísticamente significativas (Field, 2009).

Tabla 3: Competencia: Correlación Elemento-Total Corregidas

\begin{tabular}{ccccc}
\hline Estadísticos total-elemento & & & \\
\hline & Media de la escala si & Varianza de la & Correlación & Alfa de Cronbach si \\
se elimina el & escala si se elimina & elemento-total & se elimina el \\
& elemento & el elemento & corregida & elemento \\
\hline N3 & 18.90 & 4.563 & $\mathbf{. 6 2 0}$ & .720 \\
N6 & 19.19 & 3.755 & .691 & .669 \\
N9 & 19.12 & 4.250 & .538 & .752 \\
N12 & 19.06 & 4.096 & $\mathbf{. 5 2 3}$ & .764
\end{tabular}


En la sub-escala de Relación (Ver tabla 4) se obtuvo un coeficiente de consistencia Alfa de Cronbach de .744 y las correlaciones elementos total corregida fueron de .539 a .360, los cuales son mayores a .30, siendo así, estadísticamente significativas (Field, 2009).

Tabla 4 : Relación: Correlación Elemento-Total Corregidas

\begin{tabular}{|c|c|c|c|c|}
\hline \multicolumn{5}{|c|}{ Estadísticos total-elemento } \\
\hline & Media de la escala si & Varianza de la & Correlación & Alfa de Cronbach si \\
\hline & se elimina el & escala si se elimina & elemento-total & se elimina el \\
\hline & elemento & el elemento & corregida & elemento \\
\hline $\mathrm{N} 2$ & 17.59 & 5.198 & .539 & .685 \\
\hline N5 & 17.65 & 5.054 & .661 & .620 \\
\hline N8 & 17.77 & 4.838 & .618 & .638 \\
\hline N11 & 17.78 & 5.842 & .360 & .783 \\
\hline
\end{tabular}

Estos resultados permiten brindar evidencias de validez de constructo y la confiabilidad de la Escala de Satisfacción y Frustración de Necesidades Psicológicas Básicas (BPNSFS) en la muestra empleada en el presente estudio por lo cual es posible continuar con los siguientes análisis.

Evidencias de validez del cuestionario problemas en el trabajo (PAW).

\section{Primer análisis.}

Para demostrar la validez del constructo del instrumento Cuestionario Problemas en el Trabajo (PAW), se realizó un análisis de componentes principales con rotación Varimax. Se utilizó la medida de adecuación muestral Kaiser - Meyer - Olkin (KMO) para contrastar las 
correlaciones parciales entre las variables; en otras palabras, nos indica la proporción de la varianza que tienen en común las variables analizadas. Los valores del KMO van del 0 al 1 , Kaiser (1974) recomienda aceptar valores mayores a 0.5, ya que los valores menores a éste pueden llevar a recolectar más datos o re evaluar que variables a incluir.

También se utilizó la prueba de esfericidad de Bartlett, la cual busca contrastar la hipótesis nula de que la matriz de correlaciones es igual a una matriz de identidad. Con esta prueba se busca una multicolinealidad, por lo tanto, es necesario rechazar la hipótesis nula y aceptar la hipótesis alternativa de que la matriz es distinta a una matriz de identidad, y por ende hay un nivel suficiente de multicolinealidad entre las variables. Si el valor es superior a .05 entonces no podremos rechazar la hipótesis nula de esfericidad (Field, 2009).

El análisis de la adecuación de la muestra dio como resultado un KMO igual a 0.67, lo cual es un valor aceptado e indica una buena adecuación de los datos. La prueba de esfericidad de Bartlett $(\mathrm{X} 2=1287.284, \mathrm{gl}=496, \mathrm{p}<.001)$ tiene una significación menor a .05 , por lo que se puede rechazar dicha hipótesis nula y considerar que existe un nivel de multicolinealidad entre las variables. En este proceso, se realizaron varios análisis de componentes principales, los que se describen a continuación.

Como resultado del primer análisis se encontraron 10 componentes que explicaron el $68.03 \%$ de la varianza total. Sin embargo, cabe señalar que esta solución dista de la solución original del instrumento de Problemas en el Trabajo, el cual tendría que arrojar cuatro componentes: Alto apoyo a la autonomía, alto apoyo al control, moderado apoyo a la autonomía y moderado apoyo al control. 


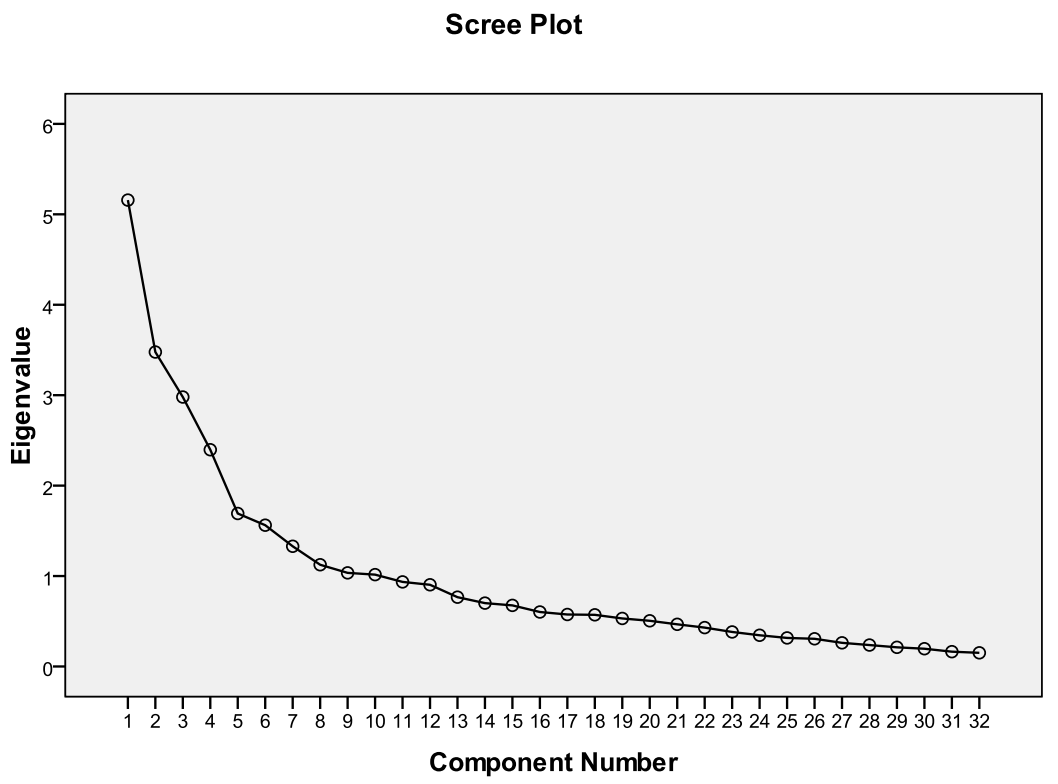

Gráfico 1: Gráfico de Sedimentación de Cattel sugiere retener 4 componentes

Las cargas factoriales del análisis de 10 componentes se muestran en la tabla 2. En esta tabla puede verse que el componente 1 está conformado por los ítems 14, 3, 24 y 26, de los cuales 14 y 24 pertenecen a la escala de MC, 3 a la escala AC y 26 a MA. El componente 2 está conformado por los ítems $13,2,23,11,28,18$ y 5, de los cuales todos pertenecen a la escala de AA. El componente 3 está conformado por los ítems 32, 27, 1, 29, 15, 8, 6 y 22, de los cuales 32, 27, 8 y 22 pertenecen a la escala AC, los ítems 1 y 6 pertenecen a la escala de MC, el ítem 29 pertenece a la escala AA, y el ítem 15 pertenece a la escala de MA. El componente 4 está conformado por los ítems 10, 7, 31, 21 y 4, los cuales pertenecen a la escala de MA. El componente 5 está conformado por los ítems 19 y 20, el ítem 19 pertenece a la escala de MC y el ítem 20 a la escala de MA. El componente 6 está conformado por los ítems 25 y 30, los cuales pertenecen a la escala de MC. El componente 7 está conformado por los ítems 16 y 12, en el cual el ítem 16 pertenece a la escala AC y el ítem 12 a la escala de MC. El componente 8 está compuesto por el ítem 9 que pertenece a la escala de AC. El componente 9 no tiene ningún ítem; y por último el componente 10 está conformado por el ítem 17 que pertenece a la escala de AC (Ver tabla 5). 
Tabla 5 : Matriz de componentes Rotados- Primer análisis

\begin{tabular}{|c|c|c|c|c|c|c|c|c|c|c|}
\hline & Compc & nentes & & & & & & & & \\
\hline & 1 & 2 & 3 & 4 & 5 & 6 & 7 & 8 & 9 & 10 \\
\hline P14 & .765 & & .252 & & & & & & & \\
\hline P3 & .747 & & & & & & & & & \\
\hline P24 & .642 & & & & & & & & & .222 \\
\hline P26 & .512 & & & .506 & & & & & & -.218 \\
\hline P13 & & .734 & & & & & -.228 & & .232 & \\
\hline $\mathrm{P} 2$ & & .717 & & & & & & & & \\
\hline P23 & & .701 & & & & & & -.243 & & \\
\hline P11 & & .647 & & & -.300 & .213 & & & & -.298 \\
\hline P28 & -.252 & .570 & .270 & & & & .282 & .216 & -.358 & \\
\hline P18 & & .562 & & & -.415 & & .213 & & & -.284 \\
\hline P32 & & & .792 & & & .203 & & & & \\
\hline P27 & & & .662 & & & .242 & & -.229 & & .358 \\
\hline P1 & & & .523 & & & & & .231 & .385 & \\
\hline P29 & -.335 & .358 & .519 & & -.201 & -.241 & & & & .223 \\
\hline P15 & & & .518 & .472 & -.329 & & & & & \\
\hline P8 & .365 & -.206 & .496 & & .268 & -.339 & & & & \\
\hline P6 & .320 & .213 & .330 & -.228 & & & -.282 & & & \\
\hline P10 & & & & .752 & & & -.255 & & & \\
\hline P7 & & & & .739 & & -.270 & & & & \\
\hline P31 & .288 & & & .621 & & & .263 & & & -.253 \\
\hline
\end{tabular}




\begin{tabular}{|c|c|c|c|c|c|c|c|c|c|}
\hline P21 & & .324 & .516 & & -.219 & & & .494 & \\
\hline P5 & .378 & & & -.751 & & & & & \\
\hline $\begin{array}{ll}\mathrm{P} 19 \quad .578\end{array}$ & & & & .595 & & & & & \\
\hline .406 & & .333 & & .491 & & & -.220 & & \\
\hline .210 & & & & & .795 & & & & \\
\hline P30 & .214 & & & & .626 & .365 & .309 & & .207 \\
\hline .242 & & & & & & .816 & & & \\
\hline P12 & & .353 & & & -.331 & .356 & & .290 & .330 \\
\hline .224 & & & & & & & .842 & & \\
\hline .269 & & .443 & & & & & -.481 & .432 & \\
\hline P4 & & & .422 & & & & & -.651 & \\
\hline .362 & -.232 & & & & & & & & .732 \\
\hline
\end{tabular}

Como se señaló, el resultado de este análisis no coincide con la estructura original del instrumento, ya que cuatro de los componentes $(1,3,5$ y 7$)$ tienen ítems con cargas factoriales compartidas, es decir los ítems “cargan” en componentes donde no deberían de estar. En otras palabras, estos cuatro componentes cargan ítems de las cuatro sub-escalas de la prueba, cuando en realidad, cada componente debe de agrupar los ítems de una misma sub-escala.

En conclusión, los resultados arrojados en el primer análisis no permitieron observar con claridad las cuatro sub-escalas que la prueba original proponía por lo cual se considero realizar otro análisis para poder identificarlos con claridad pues en el primer análisis era difícil poder diferenciar los componentes. 


\section{Segundo análisis.}

El análisis de la adecuación de la muestra dio como resultado un Kaiser - Meyer Olkin (KMO) igual a 0.67, el cual es un valor significativo, indica una buena adecuación de los datos. La prueba de esfericidad de Bartlett $(\mathrm{X} 2=1287.284, \mathrm{gl}=496, p<.001)$, tiene un valor menor a .05 , por lo que se puede rechazar dicha hipótesis nula y considerar que existe un nivel de multicolinealidad entre las variables.

Para este segundo análisis, se retuvo 4 componentes, de acuerdo con las áreas del instrumento original (Alto apoyo a la autonomía, moderado apoyo a la autonomía, alto apoyo al control, moderado apoyo al control). Se encontró que los 4 componentes explicaron el 43.78\% de la varianza total. El primer componente obtuvo un autovalor de 3.915 y explicó el $22.29 \%$ de la varianza, el segundo componente obtuvo un autovalor de 3.781 y explicó el $11.816 \%$ de la varianza, el tercer componente obtuvo un autovalor de 3.547 y explicó el 11.084 $\%$ de la varianza, y el cuarto componente obtuvo un autovalor de 2.768 y explicó el $8.649 \%$ de la varianza.

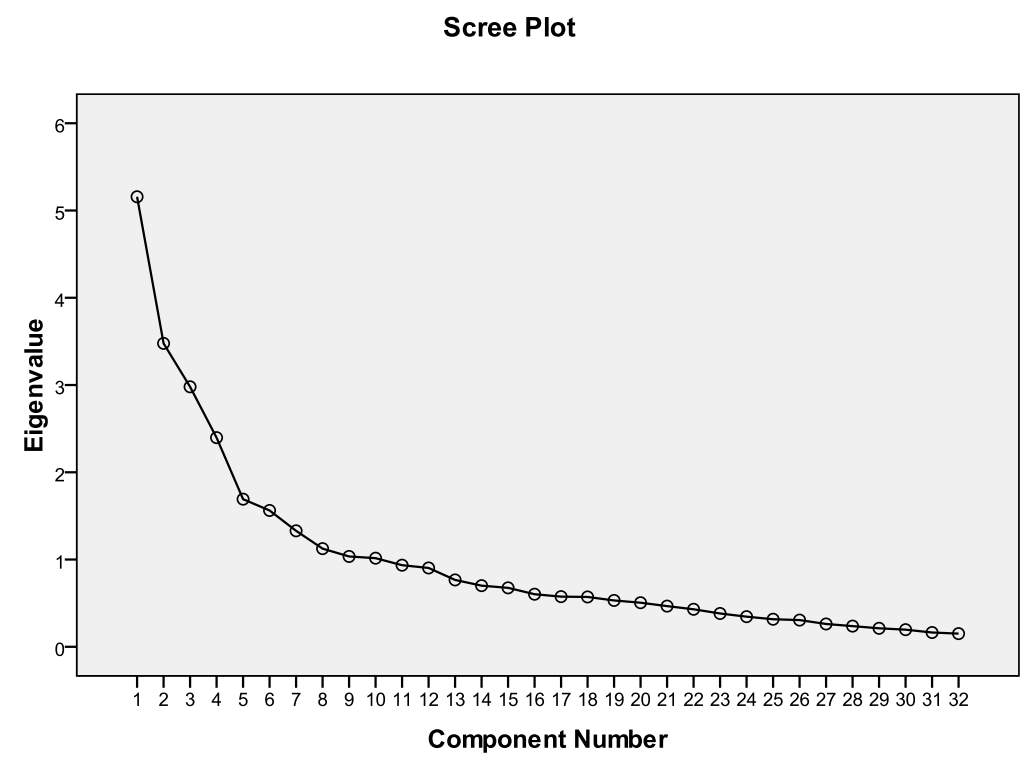

Gráfico 2: Gráfico de Sedimentación de Cattel sugiere retener también 4 componentes. 
Se analizaron las cargas factoriales de los 4 componentes, en base a la tabla de la Matriz de los Componentes Rotados de la tabla 5.

Se puede ver (tabla 6) que el componente 1 está conformado por los ítems 27, 22, 32, $1,12,15,8,21,29,17,20$ y 6 , de los cuales los ítems $27,22,32,8,17$ pertenecen a la escala $\mathrm{AC}, \operatorname{los}$ ítems 1,12 y 6 pertenecen a la sub-escala MC, los ítems 15,21 y 20 pertenecen a la sub-escala MA, y el ítem 29 pertenece a la escala AA. El componente 2 está conformado por los ítems $11,2,18,13,28,5,23$ y 30, de los cuales los ítems 11, 2, 18, 13, 28, 5 y 23 pertenecen a la sub-escala de AA, y el ítem 30 pertenece a la escala MC. El componente 3 está compuesto por los ítems 14, 19, 3, 24, 9, 25 y 16, de los cuales los ítems 14, 19, 24 y 25 pertenecen a la sub-escala de MC, y los ítems 3, 9 y 16 pertenecen a la sub-escala de AC. El componente 4 está conformado por los ítems 31, 7, 10, 4 y 26 los cuales todos pertenecen a la escala de MC. (Ver Tabla 6)

Tabla 6 : Matriz de Componentes Rotados - Segundo Análisis

\begin{tabular}{cccc}
\hline \multicolumn{4}{l}{ Componentes } \\
\hline 1 & 2 & 3 & 4
\end{tabular}

P27: AC .724

P22: AC .715

P32: AC $\mathbf{. 6 6 5}$

$\mathrm{P} 1: \mathrm{MC} \quad \mathbf{5 7 2}$

P12: MC .572

P15: MA .510

P8: AC $\quad \mathbf{. 5 0 7} \quad-.313 \quad .225$ 


\begin{tabular}{|c|c|c|c|c|}
\hline P29: AA & .466 & .407 & -.424 & \\
\hline P17: AC & .458 & -.322 & .325 & \\
\hline P20: MA & .429 & & .422 & .261 \\
\hline P6: MC & .242 & & .235 & -.223 \\
\hline P11: AA & & .772 & & \\
\hline P2: AA & & .749 & & \\
\hline P18: AA & & .693 & & \\
\hline P13: AA & & .607 & & \\
\hline P28: AA & & .603 & & \\
\hline P5: AA & & .576 & & \\
\hline P23: AA & & .531 & & \\
\hline P30: MC & & .378 & .324 & \\
\hline P14: MC & .279 & & .700 & \\
\hline P19:MC & & -.209 & .698 & .249 \\
\hline P3:AC & & -.258 & .673 & \\
\hline P24: MC & & & .588 & \\
\hline P9: AC & & & .423 & \\
\hline P25: MC & & .211 & .423 & \\
\hline P16: AC & & & .381 & \\
\hline P31: MA & & & .322 & .693 \\
\hline P7: MA & .257 & & -.280 & .691 \\
\hline P10: MA & & & & .658 \\
\hline
\end{tabular}


El resultado de este análisis no coincide con la estructura original del instrumento de Problemas en el Trabajo (PAW). Tres componentes, el 1, 2 y 3, presentan ítems con cargas factoriales compartidas, es decir los ítems cargan en componentes en donde no deberían de estar.

De esta manera, el análisis realizado demostró que existían diversas cargas factoriales compartidas lo cual no permitía identificar con claridad los cuatro componentes que explica la prueba original por lo que se realizó un tercer análisis basándose en la existencia de solo dos componentes: Alto Apoyo a la Autonomía y Alto apoyo al control Control.

\section{Tercer análisis.}

El análisis de la adecuación de la muestra dio como resultado un Kaiser - Meyer Olkin (KMO) igual a 0.708, el cual es un valor significativo, indica una buena adecuación de los datos. La prueba de esfericidad de Bartlett $(\mathrm{X} 2=494.698, \mathrm{gl}=120, \mathrm{p}<0.01)$, tiene un valor menor a .05 , por lo que se puede rechazar dicha hipótesis nula y considerar que existe un nivel de multicolinealidad entre las variables. Estos resultados permiten continuar con este tipo de análisis.

En este análisis de componentes principales, se retuvo 2 componentes, los cuales explicaron el $39.19 \%$ de la varianza total. El primer componente obtuvo un autovalor de 3.52 y explicó el $21.99 \%$ de la varianza. El segundo componente obtuvo un autovalor de 2.75 y explicó el $17.20 \%$ de la varianza. 


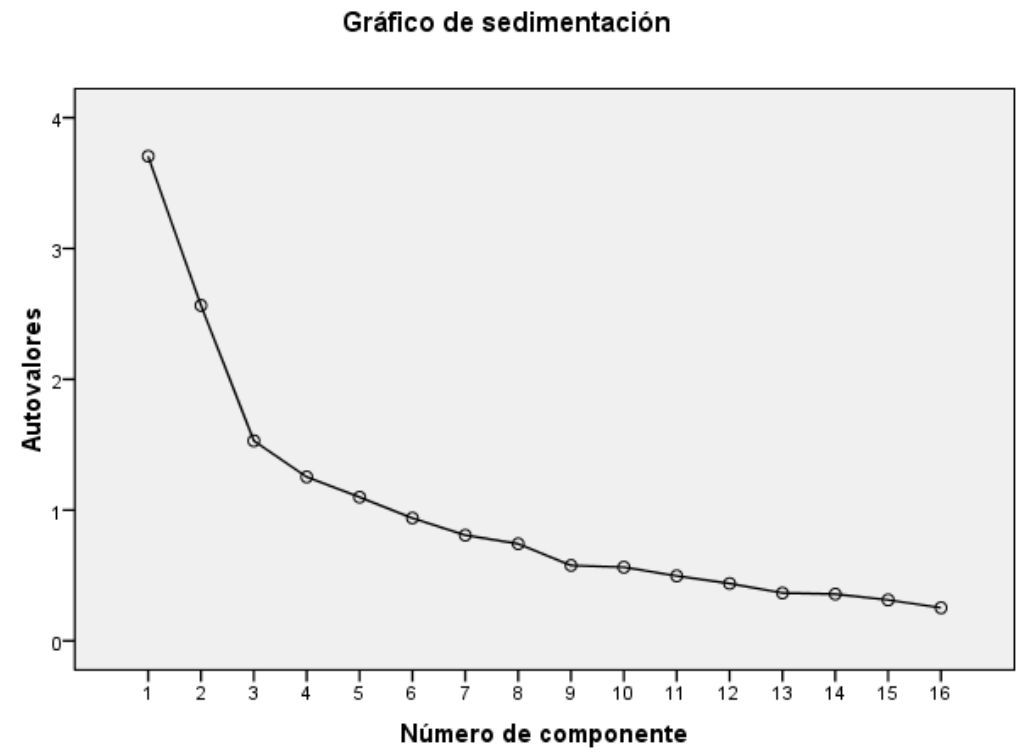

Gráfico 3: Gráfico de Sedimentación de Cattel sugiere retener también 2 componentes.

Se analizaron las cargas factoriales de los 2 componentes, basándonos en la tabla de la Matriz de los Componentes Rotados de la tabla 6.

Tabla 7 : Validez: Matriz de componentes Rotados- Tercer Análisis

\begin{tabular}{lll}
\hline \multicolumn{3}{c}{ Componentes } \\
\hline & $1(\mathrm{AA})$ & $2(\mathrm{AC})$ \\
\hline P2: AA & $\mathbf{. 7 4 3}$ & -.024 \\
P11: AA & $\mathbf{. 7 3 4}$ & -.191 \\
P18: AA & $\mathbf{. 6 6 4}$ & -.251 \\
P28: AA & $\mathbf{. 6 5 5}$ & .036 \\
P13: AA & $\mathbf{. 6 1 3}$ & .006 \\
P5: AA & $\mathbf{. 5 6 6}$ & .011 \\
P29: AA & $\mathbf{. 5 4 4}$ & .400 \\
P23: AA & $\mathbf{. 5 3 7}$ & -.134
\end{tabular}




$\begin{array}{lll}\text { P3: AC } & -.323 & \mathbf{. 2 3 9} \\ \text { P27: AC } & -.008 & \mathbf{. 7 7 4} \\ \text { P22: AC } & -.021 & \mathbf{. 7 2 3} \\ \text { P32: AC } & .183 & \mathbf{. 7 1 7} \\ \text { P17: AC } & -.298 & \mathbf{. 5 9 4} \\ \text { P8: AC } & -.221 & \mathbf{. 5 8 6} \\ \text { P16: AC } & .009 & \mathbf{. 2 6 0} \\ \text { P9: AC } & \mathbf{. 0 3 9} & -.128\end{array}$

Se puede ver (tabla 7) que el componente 1 está conformado por los ítems 2, 11, 18, $28,13,5,29,23$ y 9 , de los cuales $2,11,18,28,13,5,29$ y 23 pertenecen a la sub-escala de AA, y el ítem 9 pertenece a la escala de AC. El componente 2 está conformado por los ítems $3,27,22,32,17,8$ y 16, los cuales todos pertenecen a la sub-escala de AC.

De acuerdo al análisis, el primer componente presenta ítems con cargas factoriales compartidas. Es necesario realizar otro análisis de los componentes principales, eliminando el ítem 9 porque tiene una carga baja y negativa donde debería cargar de manera positiva, que vendría a ser en el componente 2 (AC). Es necesario eliminar también el ítem 3 porque presenta una carga factorial baja (.24) en el área donde debería cargar (componente 2) mientras que es de -.32 en el componente 1 (escala de AA). También es necesario eliminar el ítem 16 porque tiene una carga factorial baja (.26) en el componente 2 , que es a la escala que pertenece. Asimismo, se requiere eliminar el ítem 29 porque presenta altas cargas factoriales altas en ambos componentes. Si bien, la carga es más alta en el componente 1 (.54), que es el esperado, es también alta en el otro componente (.40) por lo cual no es un ítem que ayude a discriminar 
con claridad el estilo de motivación. Se realizó un análisis adicional sin considerar los ítems mencionados, pero respetando las dimensiones de Alto apoyo a la Autonomía y alto control.

\section{Cuarto análisis.}

A continuación, se realiza un cuarto análisis de los componentes principales solicitando la retención de 2 componentes pero eliminando los ítems 9, 3, 16 y 29 de acuerdo con los resultados del análisis anterior. Los ítems 11, 2, 18, 13, 28, 5 y 23 se encuentran en el componente 1 (AA) y los ítems 27, 22, 32, 8 y 17 se encuentran en el componente 2 (AC).

El análisis de la adecuación de la muestra dio como resultado un Kaiser - Meyer Olkin (KMO) igual a 0.764, el cual es un valor significativo, indica una buena adecuación de los datos. La prueba de esfericidad de Bartlett $(\mathrm{X} 2=353.298, \mathrm{gl}=66, p<.01)$, tiene un valor menor a .05 , por lo que se puede rechazar dicha hipótesis nula y considerar que existe un nivel de multicolinealidad entre las variables.

Estos 2 componentes explicaron el $47.999 \%$ de la varianza total. El primer componente obtuvo un autovalor de 3.234 y explicó el $26.949 \%$ de la varianza. El segundo componente obtuvo un autovalor de 2.526 y explicó el $21.501 \%$ de la varianza.

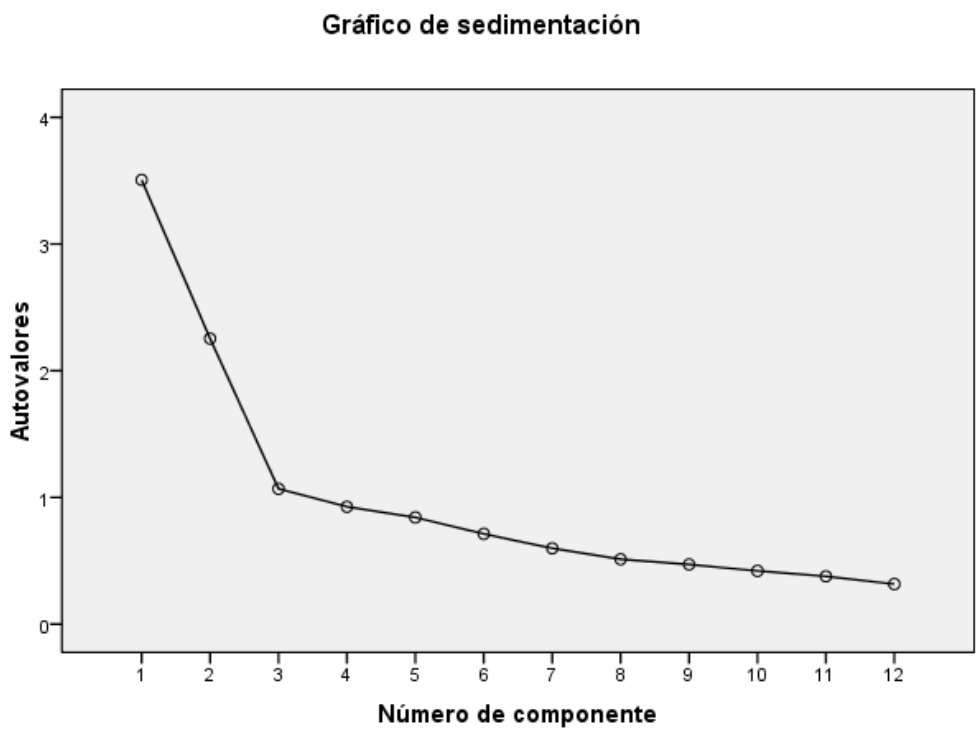


Gráfico 4: Gráfico de Sedimentación de Cattel sugiere retener también 2 componentes.

Se analizarán las cargas factoriales de los 2 componentes, basándonos en la tabla 7. Tal como se observa en la tabla 8 , el componente 1 está conformado por los ítems $11,2,18,13$, 28, 5 y 23 los cuales pertenecen a la sub-escala AA. El componente 2 está conformado por los ítems 27, 22. 32, 8, 17 los cuales todos pertenecen a la sub-escala de AC.

Tabla 8 : Matriz de componentes Rotados

\begin{tabular}{|c|c|}
\hline & Componentes \\
\hline & 1:AA 2: AC \\
\hline P11:AA & .773 \\
\hline P2:AA & .757 \\
\hline P18: AA & .684 \\
\hline P13: AA & .661 \\
\hline P28: AA & .600 \\
\hline P5: AA & .581 \\
\hline P23: AA & .558 \\
\hline P27: AC & .788 \\
\hline P22: AC & .754 \\
\hline P32: AC & .732 \\
\hline P8: AC & .600 \\
\hline P17: AC & .573 \\
\hline
\end{tabular}


Con este cuarto análisis se pueden brindar evidencias de validez para el Cuestionario de Problemas en el Trabajo (PAW) en la muestra estudiada, que mide lo que realmente está diseñado para medir. Esto se puede ver en la Tabla 8 donde los ítems cargan en las sub-escalas correctas y esperadas, según el cuestionario, y con cargas mayores a .558 , siendo un valor significativo.

\section{Confiabilidad del cuestionario problemas en el trabajo (PAW).}

Luego de realizar el análisis de los componentes principales, se analizó la confiabilidad de cada uno de los componentes de la prueba, esto es de las sub-escalas de AA y AC. Para determinar las evidencias de confiabilidad se estudió la consistencia interna (coeficiente Alfa de Cronbach), ya que permite estimar la confiabilidad del instrumento y que el conjunto de ítems miden un mismo constructo y que están altamente correlacionadas entre sí (Cronbach, 1951). También, se utilizó la correlación elemento-total corregida para poder analizar la correlación lineal entre el ítem y la puntuación total y así señalar el grado y dirección de esta relación, las correlaciones a partir de .30 son estadísticamente significativas (Field, 2009).

En la sub-escala de AA (Ver tabla 9) se obtuvo un coeficiente de consistencia Alfa de Cronbach de .771 el cual es un buen valor, y las correlaciones elementos total corregida se encontraron entre .423 y .642 , los cuales son mayores a 0.30 , siendo así, estadísticamente significativas (Field, 2009). 
Tabla 9 : Alto Apoyo a la Autonomía: Correlaciones Elemento-Total Corregidas

Estadísticos total-elemento

\begin{tabular}{lcccc}
\hline & $\begin{array}{c}\text { Media de la escala si } \\
\text { se elimina el }\end{array}$ & $\begin{array}{c}\text { Varianza de la } \\
\text { escala si se elimina }\end{array}$ & Correlación & Alfa de Cronbach si \\
& elemento & el elemento & corregida & elemento \\
\hline P11 & 34.66 & 23.656 &. $\mathbf{6 4 2}$ & .725 \\
P2 & 35.03 & 20.779 & .623 & .713 \\
P18 & 34.82 & 23.435 & .567 & .732 \\
P13 & 35.25 & 23.398 & .500 & .742 \\
P28 & 35.00 & 23.064 & .428 & .756 \\
P5 & 35.70 & 21.074 & .409 & .774 \\
P23 & 35.55 & 23.498 & .423 & .756 \\
\hline
\end{tabular}

En la sub-escala de AC (Ver tabla 10) se obtuvo un coeficiente de consistencia Alfa de Cronbach de .734 el cual es un buen valor y las correlaciones elementos total corregida se encontraron entre .406 y .620, los cuales son mayores a .30, siendo así estadísticamente significativas (Field, 2009). 
Tabla 10 : Alto Apoyo al Control: Correlaciones Elemento-Total Corregidas

Estadísticos total-elemento

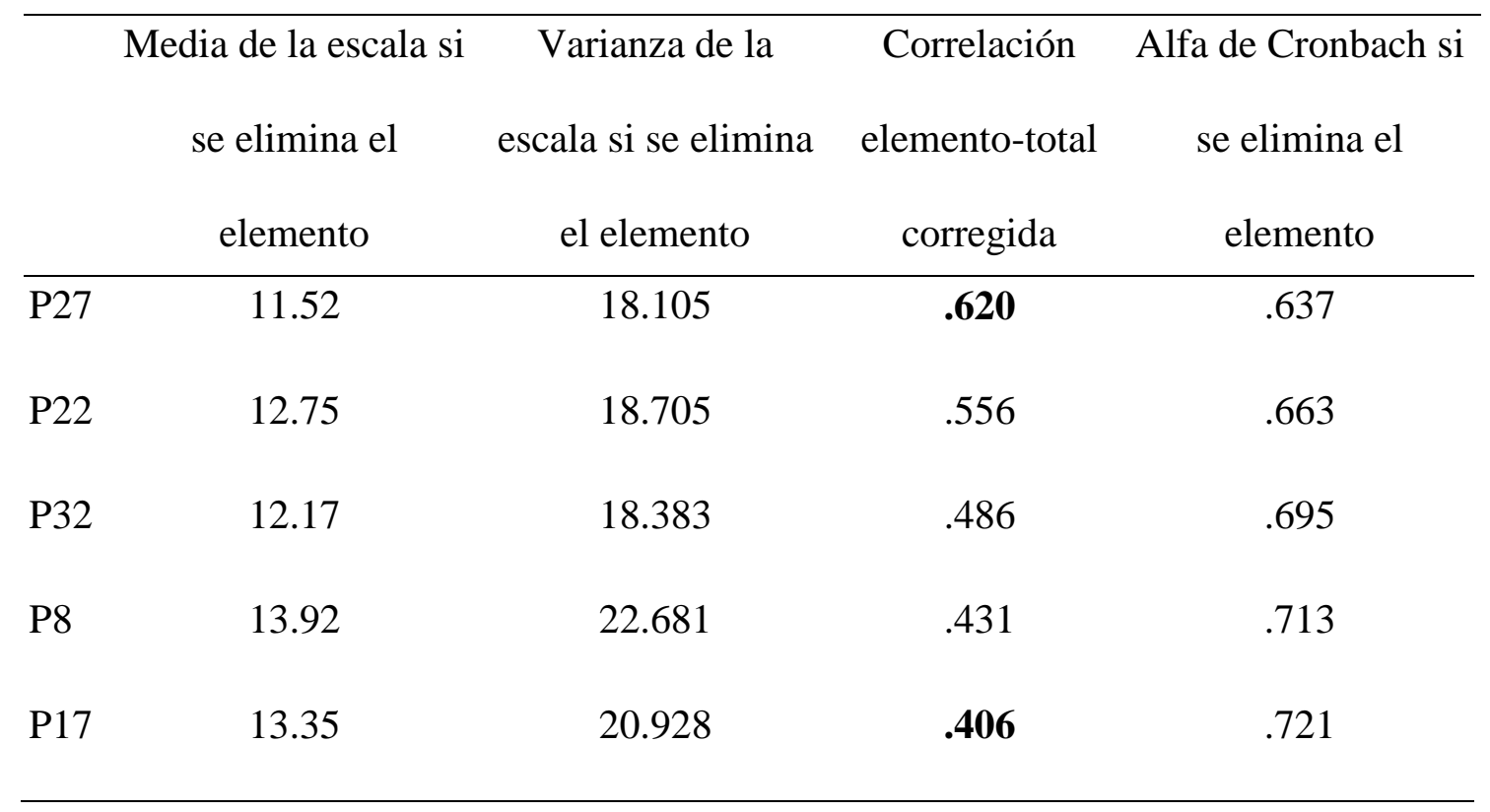

Estos resultados obtenidos han sido significativos los cuales han permitido brindar evidencias de validez de constructo y de confiabilidad del Cuestionario de Problemas en el Trabajo y por lo tanto es posible continuar con los análisis siguientes. 


\section{Análisis Descriptivo}

En la Tabla 11 se puede observar las medias, la cual indica el promedio de los valores de cada su-escala y las desviaciones estándar, que indican el grado de dispersión de cada subescala respecto a su media. La sub-escala de AA obtuvo una media de 5.85 y una desviación estándar de .779. La sub-escala de AC obtuvo una media de 3.18 y una desviación estándar de 1.07. La sub-escala de Autonomía obtuvo una media de 5.82 y una desviación estándar de .748. La sub-escala de Competencia obtuvo una media de 6.35 y una desviación estándar de .656. La sub-escala de Relación obtuvo una media de 6.02 y una desviación estándar de .688.

Tabla 11 : Descriptivos Estadísticos de las variables Estadísticos descriptivos

N Mínimo Máxim Media Desv. típ.

\begin{tabular}{lccccc}
\hline Alta apoyo a la Autonomía & 110 & 2.71 & 7.00 & 5.8571 & .77934 \\
Alto apoyo al Control & 110 & 1.00 & 5.60 & 3.1855 & 1.07608 \\
Autonomía & 110 & 3.50 & 7.00 & 5.8205 & .74895 \\
Competencia & 111 & 3.75 & 7.00 & 6.3559 & .65674 \\
Relación & 110 & 3.50 & 7.00 & 6.0205 & .68831 \\
& & & & & \\
\hline
\end{tabular}




\section{Prueba de Normalidad}

Además, se exploró si los datos estaban distribuidos normalmente mediante la prueba de Kolmogorov-Smirnov, la cual permite determinar la medición del grado de concordancia que existe entre la distribución de la muestra, que es mayor a 50 personas, y una distribución teórico específica. Se puede observar los siguientes resultados: AA ( $\mathrm{Z}=1.169, \mathrm{p}=.130), \mathrm{AC}$ $(\mathrm{Z}=.924, \mathrm{p}=.360)$, Autonomía $(\mathrm{Z}=1.514, \mathrm{p}=.020)$, Competencia $(\mathrm{Z}=1.721, \mathrm{p}=.005)$, Relación $(\mathrm{Z}=1.687, \mathrm{p}=.007)$. Estos resultados indican que la distribución no es normal para las variables de autonomía, competencia y relación y por eso se utilizará la correlación de Spearman para los análisis de la relación entre variables. El coeficiente de correlación Spearman es una medida de la asociación entre dos variables aleatorias. 


\section{Correlaciones entre las Variables}

Tabla 12: Relación entre las variables estudiadas

\begin{tabular}{|c|c|c|c|c|}
\hline & Alta Autonomía & Alto Control & Autonomía & Competencia \\
\hline Alta autonomía & - & & & \\
\hline Alto control & $-.16^{*}$ & & & \\
\hline Autonomía & $.29 * *$ & .03 & & \\
\hline Competencia & $.30 * *$ & -.01 & $.53 * * *$ & \\
\hline Relación & $.35 * * *$ & .04 & $.52 * * *$ & $.69 * * *$ \\
\hline
\end{tabular}

Tal como se observa en la Tabla 12, se encontró que la sub-escala alto apoyo a la autonomía correlaciona positivamente y significativamente con las tres necesidades psicológicas básicas: autonomía $\left(.29^{* *}, p<.01\right)$, competencia $\left(.30^{* *}, p<.01\right)$ y relación $\left(.35^{* * *}, p<0.001\right)$. Esto quiere decir que la investigación indica que los supervisores que tienen un estilo de motivación que apoya la autonomía tienen satisfechas las tres necesidades psicológicas básicas de autonomía, competencia y relación y viceversa. Esto es, mientras mayor sea el nivel de satisfacción de las necesidades psicológicas básicas del supervisor, éste estará más propenso a utilizar el estilo motivacional de apoyo a la autonomía con sus trabajadores/empleados.

Los resultados arrojan claramente una relación positiva y significativa más fuerte de la sub-escala de AA con la necesidad psicológica básica de relación $\left(.35^{* * *}, p<.001\right)$, lo cual quiere decir que los supervisores con un estilo de alto apoyo a la autonomía, tienen la necesidad psicológica básica de relación satisfecha en mayor grado. 
También se encontró que las tres necesidades psicológicas básicas correlacionan positiva y significativamente entre ellas: autonomía y competencia $(.53 * * *, p<.001)$, autonomía y relación $\left(.52^{* * *}, p<.001\right)$ y donde hubo una correlación más fuerte fue entre las necesidades de competencia y relación $\left(.69^{* * *}, p<.001\right)$.

Tal como se espera, existe una correlación negativa y un tanto significativa entre el alto apoyo a la autonomía y el alto apoyo al control (-.16*, $p<.05)$. Es decir, mientras un supervisor utilice un estilo de motivación con un mayor grado de apoyo a la autonomía, su estilo de motivación de control será menor.

Por otro lado, no existe correlación entre un estilo de motivación de apoyo al control y la satisfacción de necesidades psicológicas básicas. 


\section{Discusión}

El objetivo principal de esta investigación identificó que existe una relación entre las dos variables estudiadas. Existe una relación positiva y significativa de la satisfacción de las necesidades psicológicas básicas y un estilo motivacional de apoyo a la autonomía en los supervisores. En otra palabras, a mayor nivel de la satisfacción de las necesidades básicas del supervisor, éste tendrá mayor inclinación a un estilo motivacional favorable con sus trabajadores. Es importante considerar el ámbito organizacional donde labora el supervisor, ya que puede favorecer a la satisfacción de sus necesidades.

Según la teoría, los contextos sociales que facilitan la satisfacción de estas tres necesidades psicológicas básicas, son aquellas que apoyan la actividad personal de cada individuo, motivan intrínsecamente a sus empleados y logran resultados positivos dentro del desarrollo psicológico y de la conducta de la persona (Deci \& Ryan, 2012b; Ryan \& Deci, 2000). Entonces, de acuerdo a la teoría y los resultados, un supervisor que se encuentra en un ambiente laboral que favorece la satisfacción de sus propias necesidades psicológicas, mayor será la tendencia a utilizar un estilo motivacional de apoyo a la autonomía, y evitará las practicas de un estilo motivacional controlador.

Según la teoría, dentro de un ambiente laboral existen factores que promueven la autodeterminación, algunas de ellas son la capacidad de elección, un feedback no controlador, la aceptación y reconocimiento de la perspectiva del otro (Ryan, 1982; Zuckerman, Porac, Lathin \& Deci, 1978). Estos factores en conjunto, constituyen las características de un estilo de motivación de apoyo a la autonomía. Por ende, la acción de apoyar la autonomía refleja un sentido de autodeterminación, que influye positivamente en la creatividad, el aprendizaje conceptual, el tono emocional y la autoestima (Deci et al., 1989). De esta manera, se podría afirmar que la acción de apoyar la autonomía es una experiencia positiva con la cual el supervisor satisface sus necesidades psicológicas básicas, logrando un sentido de auto- 
determinación. De esta manera, tenderá a utilizar un estilo motivacional favorable con sus empleados, logrando satisfacer sus necesidades psicológicas básicas generando un impacto positivo en el desarrollo y bienestar emocional de sus empleados (Ryan \& Deci, 2002).

En cuanto al grado de satisfacción de las tres necesidades psicológicas básicas de los supervisores, se encontró que hubo una relación positiva y significativa entre ellas, pero la correlación más fuerte fue entre la necesidad de competencia y la de relación.

En un estudio realizado por Hofer y Busch (2011) se encontró que la satisfacción de las necesidades de competencia y relación están asociadas con niveles altos de satisfacción en el trabajo y en las relaciones interpersonales. La fuerte relación entre la necesidad de competencia y relación en la presente investigación, señala que los supervisores que se sienten confiados y competentes en su trabajo tienden a tener una mejor relación interpersonal con sus compañeros de trabajo y viceversa. Además, al ser una correlación positiva, también se puede afirmar que los supervisores que se valoran las relaciones interpersonales, buscan situaciones donde las personas satisfagan también su necesidad psicológica básica de competencia y puedan poner en práctica sus capacidades, habilidades y desafiarse a sí mismos.

Los resultados también indicaron que aquellos supervisores con un estilo motivacional de apoyo a la autonomía tenían satisfecha la necesidad de relación en mayor grado. Entonces se puede señalar que los supervisores con un estilo de apoyo a la autonomía son personas que buscan relacionarse de manera real e íntima con los demás. Buscan sentirse conectados con el resto y disfrutan pertenecer a un grupo de compañeros y pares, en el que se cuiden unos con otros (Baumeister \& Leary, 1995; Bowlby, 1979; Harlow, 1958; Ryan, 1995). Además, la Teoría de la Auto-Determinación, y la Teoría de tres factores de McClelland, consideraran que las relaciones interpersonales favorables son uno de los factores principales para lograr la motivación. La necesidad de relación y afiliación, de acuerdo a cada teoría, son consideradas necesidades universales, es decir todas las personas tienen estas necesidades en mayor o menor 
grado, independientemente del género, edad o cultura, pero pueden estar satisfechas en diferentes grados (Chen et al., 2015; Uduji \& Ankeli, 2013).

Una de las características principales de un estilo de motivación de apoyo a la autonomía es la relación dialéctica que existe entre el supervisor y su empleado, lo cual permite al supervisor identificar y desarrollar recursos internos para utilizar durante el desarrollo motivacional de sus trabajadores (Deci \&Ryan, 1985b; Gagné \& Deci 2005; Hardré \& Reeve, 2009). Junto con los resultados obtenidos, podemos concluir que adoptar un estilo de apoyo a la autonomía, requiere poseer habilidades interpersonales, como, por ejemplo, tomar la perspectiva de la otra persona, lo cual influye y promueve la satisfacción de la necesidad básica de relación.

Existen ventajas dentro del ámbito empresarial de contar con empleados que utilicen un estilo motivacional que apoya la autonomía de sus empleados. Algunos beneficios para las empresas de contar con líderes que tienen un estilo de apoyo a la autonomía o que son percibidos por sus subordinados de esa manera, son los siguientes: identificación con la organización, mejora en el performance de los empleados (Gillet et al., 2013); actitudes positivas, compromiso con la organización, incremento de la motivación autónoma y bienestar psicológico (Baard et al., 2004; Deci et al., 2001; Gagné \& Deci, 2005). Por otro lado, se previenen las renuncias, el estrés psicológico y la ideación suicida (Gillet et al., 2013; Moreau \& Mageau, 2012).

Por último, dados los beneficios expuestos anteriormente sobre la importancia del apoyo a la autonomía en las empresas, un estudio (Hardré \& Reeve, 2009) encontró que cuando los gerentes reciben un entrenamiento sobre cómo motivar la autonomía de sus empleados, ellos adoptaron dicho estilo de liderazgo y sus empleados tuvieron un grado mayor de motivación autónoma y de compromiso con el trabajo que aquellos empleados del grupo de control. Entonces, es importante considerar que un estilo de motivación de autonomía sí se 
puede llegar a enseñar en cierto grado. Con lo cual se puede recomendar que las empresas brinden y fomenten un ambiente de trabajo que genere un impacto positivo en la satisfacción de las necesidades que prevenga la frustración de estas (Gillet et al., 20123). 


\section{Capítulo 4 : Conclusiones}

El objetivo principal de esta investigación fue alcanzado al identificar que aquellos supervisores con un estilo motivacional de apoyo a la autonomía, tienen satisfechas las tres necesidades psicológicas básicas. En cuanto a los objetivos específicos, los resultados establecen que la necesidad satisfecha en mayor grado en los supervisores con un estilo de apoyo a la autonomía, es la necesidad de relación. Mientras que no existe relación entre un estilo motivacional de control de los supervisores y la satisfacción de sus necesidades psicológicas básicas.

Estos resultados son de gran utilidad para aquellos investigadores que desean analizar temas relacionados con los estilos de motivación en el ámbito laboral, las necesidades psicológicas básicas en supervisores, autodeterminación en la organización, etc. Pueden tomar como referencia los resultados obtenidos y utilizarlo como apoyo literario para resaltar la importancia que fomentar una cultura laboral que favorezca la satisfacción de las necesidades psicológicas básicas.

Además, en la presente investigación se realizó la traducción al español del Cuestionario de Problemas en el Trabajo (PAW), lo cual sirve como apoyo metodológico para aquellos que deseen evaluar el estilo de motivación de supervisores en una muestra que sólo habla español. Es importante mencionar que la validez y confiabilidad ha sido probada en esta investigación.

Esta investigación confirma que para que un supervisor tenga mayores probabilidades de poder apoyar la autonomía de sus empleados es necesario que tenga satisfechas sus necesidades psicológicas básicas. Por eso, es importante fomentar en las empresas prácticas que favorezcan dicha satisfacción. La organización debe buscar promover un clima laboral de 
bienestar, apoye la capacidad de elección, tomar consciencia de la perspectiva de los demás, brindar autonomía, evitar amenazas y comportamientos controladores. 


\section{Limitaciones de la Investigación}

Una de las limitaciones del estudio fue que no se realizó un control o un análisis diferencial sobre las variables de: la cantidad de años en el puesto de trabajo, cantidad de años con el puesto de supervisor, cantidad de personas cargo, cantidad de horas de trabajo, etc. Estas variables fueron parte del cuestionario entregado, pero en cuanto a los resultados obtenidos de la muestra, no se realizó un análisis de estas variables y estilo de motivación del supervisor. Podría existir una relación entre estas variables y el estilo de motivación utilizado por el supervisor.

Otra limitación es no tomar en consideración la cantidad de años que tiene la empresa en el mercado y si es una empresa nacional o trans-nacional, ya que podría existir una relación entre el clima laboral creado por ciertas empresas y la satisfacción de las necesidades básicas de sus empleados. En todo caso, son posibles relaciones que se pueden explorar en futuras investigaciones.

Por último, si bien la validez y confiabilidad del Cuestionario de Problemas en el Trabajo (PAW) fue comprobado, se tuvieron eliminar ítems que no cargaban en las subescalas esperadas, teniendo que realizar cuatro análisis para lograr confirmar la validez de la prueba. 


\section{Recomendaciones}

A partir de la presente investigación, se pueden realizar nuevas hipótesis para desarrollar futuros estudios. Adicional a las variables estudiadas, se podría buscar identificar la relación entre las necesidades psicológicas básicas del supervisor, el estilo de motivación que utiliza y el ambiente laboral de la empresa en la que trabajan. Además, también sería interesante estudiar la percepción que tienen los empleados sobre el estilo motivacional de sus jefes y cómo afecta el estilo utilizado en su motivación y performance en el trabajo.

Además, hubiese sido interesante conocer la relación que existe entre el estilo motivacional que utiliza un supervisor, el número de años que lleva laborando y la cantidad de capacitaciones sobre liderazgo y motivación que ha recibido.

Por último, se recomienda utilizar una muestra de mayor tamaño, ya que las estimaciones estadísticas serán más precisas y con un menor riesgo de error. 


\section{Bibliografía}

Alderfer. C. (1993). Theories Reflecting my personal experience and Life Development. The Journal of Applied Behavioral Science, 25, 351-365. doi: 10.1177/002188638902500404

Amoura, C., Berjot, S., Gillet, N., Caruana, S., \& Finez, L. (2015). Effects of autonomysupportive and controlling styles on situational autonomous motivation: Some unexpected results of the commitment procedure. Psychological Reports: Employment Psychology \& Marketing, 116(1), 1-27. doi: 10.2466/14.pr0.116k10w7

Arshadi, N., (2010). Basic need satisfaction, work motivation, and job performance in an industrial company in Iran. Procedia Social and Behavioral Sciences, 5, 1267-1272, doi:10.1016/j.sbspro.2010.07.273

Baard, P. P., Deci, E. L., \& Ryan, R. (2004). Intrinsic Need Satisfaction: A Motivational Basis of Performance and Well-Being in Two Work Settings. Journal of Applied Social Psychology, 34, 2045-2068. doi:10.1111/j.1559-1816.2004.tb02690.x

Baumeister, R. \& Leary, M. R. (1995). The need to belong: Desire for interpersonal attachments as a fundamental human motivation. Psychological Bulletin, 117, 497-529. doi: 10.1037/0033-2909.117.3.497

Black, A. E. \& Deci, E. L. (2000). Autonomous Motivation on Learning Organic Chemistry: A Self-Determination Theory Perspective. Science Education, 84(6), 740-756. doi: 10.1002/1098-237X(200011)84:6<740::AID-SCE4>3.0.CO;2-3

Bowlby, J. (1979). The making and breaking of affectional bonds. London: Tavistock.

Chen, B., Mouratidis, A., Ryan, R. M., Sheldon, K.M., Soenens, B., Van Petegem, S., Verstuyf, J., Vansteenkiste, M., Beyers, W., Boone, L., Deci, E. L., Van der Kaap-Deeder, J., Duriez, B., Lens, W., \& Matos, L. (2015). Basic psychological need satisfaction, need frustration, and need strength across four cultures. Motivation and Emotion, 36, 216-236. doi: 10.1007/s11031-014-9450-1 
Chirkov, V., Ryan, R. M., Kim, Y., \& Kaplan, U. (2003). Differentiating autonomy from individualism and independence: a self-determination theory perspective on internalization of cultural orientations and wellbeing. Journal of Personality and Social Psychology, 84, 97-109. doi: 10.1037/0022-3514.84.1.97

De Cooman, R., Stynen, D., Van den Broeck, A., Sels, L. \& De Witte, H. (2013). How job characteristics relate to need satisfaction and autonomous motivation: implications for work effort. Journal Applied of Social Psycholy, 43, 1342-1352. doi:10.1111/jasp.12143

Cronbach, L. J. (1951). Coefficient alpha and the internal structure of tests. Psychometrika, 16, 297-334. doi: 10.1007/BF02310555

DeCharms, R. (1968). Personal Causation. New York: Academic Press.

Deci, E. L. (1971). Effects of externally mediated rewards on intrinsic motivation. Journal of Personality and Social Psychology, 18, 105-115. doi: 10.1037/h0030644

Deci, E. L. (1975). Intrinsic Motivation. New York: Plenum Publishing Co.

Deci, E.L., Connell, J. P., \& Ryan, R. M. (1989). Self-Determination in a Work Organization. Journal of Applied Psychology, 74, 580-590. doi: 10.1037/0021-9010.74.4.580

Deci, E. L., Eghrari, H., Patrick, B. C., \& Leone, D. (1994). Facilitating internalization: The self-determination theory perspective. Journal of Personality, 62(1), 119-142. Recuperado de: http://selfdeterminationtheory.org/SDT/documents/1994_DeciEghrariPatLeone.pdf

Deci, E. L., Koestner, R. \& Ryan, R. M. (1999). A meta-analytic review of experiments examining the effects of extrinsic rewards on intrinsic motivation. Psychologycal Bulletin, 125, 627-668. Recuperado de https://www.rug.nl/gmw/psychology/research/onderzoek_summerschool/firststep/conte nt/papers/4.4.pdf 
Deci, E. L., Olafsen, A. H., \& Ryan, R. M. (2017). Self-determination theory in work organizations: The state of a science. Annual Review of Organizational Psychology and Organizational Behavior, 4, 19-43. doi: 10.1146/annurev-orgpsych-032516-113108

Deci, E. L., \& Ryan, R. M. (1980). The empirical exploration of intrinsic motivational processes. En L. Berkowitz (Ed.), Advances in experimental social psychology (pp. 3980). doi: 10.1016/S0065-2601(08)60130-6

Deci, E. L., \& Ryan, R. M. (1985a). Intrinsic motivation and self-determination in human behavior. New York: Plenum Press. Recuperado de: http://www.springer.com/us/book/9780306420221

Deci, E. L., \& Ryan, R. M. (1985a). The General Causality Orientations Scale: SelfDetermination in Personality. Journal of Research in Personality, 19, 109-134. doi: 10.1016/0092-6566(85)90023-6

Deci, E. L., \& Ryan, R. M. (1987) The support of autonomy and the control of behavior. Journal of Personality and Social Psychology, 53(6), 1024-1037. doi: 10.1037/00223514.53.6.1024

Deci, E. L., \& Ryan, R. M. (2008) Facilitating optimal motivation and psychological wellbeing across life's domains. Canadian Psychology, 49(1), 14-23. doi: 10.1037/07085591.49 .1 .14

Deci, E. L., \& Ryan, R. M. (2000). The "what" and "why” of goal pursuits: Human needs and the self-determination of behavior. Psychological Inquiry, 11(4), 227-268. Recuperado de: https://selfdeterminationtheory.org/SDT/documents/2000_DeciRyan_PIWhatWhy.pdf

Deci, E. L., \& Ryan, R. M. (2012a). Self-determination Theory. En P. A. M. Van Lange, A. W. Kruglanski, \& E. T. Higgins (Eds.), Handbook of Theories of Social Psychology (pp. 416-437). doi: 10.4135/9781446201022 
Deci, E. L., \& Ryan, R. M. (2012b). Motivation, personality, and development within embedded social contexts: An overview of self-determination theory. En R. M. Ryan (Ed.), Oxford handbook of human motivation (pp. 85-107). doi:10.1093/oxfordhb/9780195399820.013.0006

Deci, E. L., Ryan, R. M., Gagne, M., Leone, D., Usunov, J., \& Kornazheva, B. P. (2001). Need satisfaction, motivation, and well-being in the work organizations of a former eastern bloc country. Personality and Social Psychology Bulletin, 27(8), 930-942. Recuoerado de:

http://selfdeterminationtheory.org/SDT/documents/2001_DeciRyanGagneLeoneEtal.pd f

Deci, E. L., Ryan, R. M., \& Guay, F. (2013). Self-determination theory and actualization of human potential. En In D. McInerney, H. Marsh, R. Craven, \& F. Guay (Eds.), Theory driving research: New wave perspectives on self processes and human development (pp. 109-133). Charlotte, NC: Information Age Press.

Deci, E. L., \& Vansteenkiste, M. (2004). Self-determination theory and basic need satisfaction: Understanding human development in positive psychology. Ricerche di Psichologia, 27(1). Recuperado de: https://selfdeterminationtheory.org/SDT/documents/2004_DeciVansteenkiste_SDTand BasicNeedSatisfaction.pdf

Field, A. (2005). Discovering Statistics using SPSS. Recuperado de: http://www.soc.univ.kiev.ua/sites/default/files/library/elopen/andy-field-discoveringstatistics-using-spss-third-edition-20091.pdf

Gagné, M. (2003). The Role of Autonomy Support and Autonomy Orientation in Prosocial Behavior Engagement. Motivation and Emotion, 27(3), 199-223. doi:10.1023/A:1025007614869

Gagné, M., \& Deci, E. (2005). Self-Determination theory and work motivation. Journal of Organizational Behavior, 26, 332-362. doi: 10.1002/job.322 
Gherman, T. I., Iturbe V., J. A. \& Osorio M., D. L. (2011). La Teoría motivacional de los dos factores: un caso de estudio (Tesis de Master). Recuperado de: http://tesis.pucp.edu.pe/repositorio/handle/123456789/4555

Gillet, N., Colombat, P., Michinov, E., Pronost, AM., \& Fouquereau, E. (2013). Procedural justice, supervisor autonomy support, work satisfaction, organizational identification and job performance: the mediating role of need satisfaction and perceived organizational support. Journal of Advanced Nursing, 69, 2560-2571. doi: 10.1111/jan.12144

Gillet, N., Forest, J., Benabou, C., \& Bentein, K. (2015). The effects of organizational factors, psychological need satisfaction and thwarting, and affective commitment on workers' well-being and turnover intentions. Le Travail Humain, 78, 119-140. doi: $10.3917 /$ th.782.0119

Gillet, N., Fouquereau, E., Forest, J., Brunault, P., \& Colombat, P. (2012a). The Impact of Organizational Factors on Psychological Needs and Their Relations with Well-Being. Journal of Business and Psychology, 27, 437 - 450. doi: 10.1007/s10869-011-9253-2

Gillet, N., Gagné, M., Sauvagère, S., \& Fouquereau, E. (2012b). The role of supervisor autonomy support, organizational support, and autonomous and controlled motivation in predicting employees' satisfaction and turnover intentions. European Journal of Work and Organizational Psychology, 22, 450-460. doi:10.1080/1359432X.2012.665228

Güntert, S. T. (2015). The impact of work design, autonomy support, and strategy on employee outcomes: A differentiated perspective on self-determination at work. Motivation and Emotion (pp. 99-103). doi: 10.1007/s11031-014-9412-7

Hardré, P. L., \& Reeve, J. (2009). Training corporate managers to adopt a more autonomysupportive motivating style toward employees: an intervention study. International Journal of Training and Development, 13, 165-184. doi: 10.1111/j.14682419.2009.00325.x 
Harlow, H. F. (1958). The nature of love. American Psychologist, 13, 673-685. Recuperado de: http://psychclassics.yorku.ca/Harlow/love.htm

Hernández, R., Fernández, C., \& Baptista, P. (2006). Definición del alcance de la investigación a realizar: exploratoria, descriptiva, correlacional o explicative. En McGraw-Hill Interamericana (Eds.), Metodología de la Investigación (pp. 99-117). México: Infagon Web.

Herzberg, F. (2003). One more time: how do you motivate employees? Harvard Business Review. Recuperado de http://hbr.org/2003/01/one-more-time/ar/1

Hofer, J. \& Busch, H. (2011). Satisfying One's Needs for Competence and Relatedness: Consequent Domain-Specific Well-Being Depends on Strength of Implicit Motives. Personality and Social Psychology Bulletin, $37, \quad 1147 \quad$ - 1158 doi:10.1177/0146167211408329

Hon, A. (2011). Shaping Environments Conductive to Creativity: The Role of Intrinsic Motivation, Cornell Hospitality Quarterly, 53, 53 - 64. https://doi.org/10.1177/1938965511424725

Hu, L.T. \& Bentler, P.M. (1999). Cutoff Criteria for Fit Indexes in Covariance Structure Analysis: Conventional Criteria Versus New Alternatives. Structural Equation Modeling, 6, 1-55.

Huamaní, P. L., \& Acuña, P. (2015). Productividad Empresarial: Caso Gerencia de Infraestructura en Telefónica del Perú. Recuperado de la Universidad Mayor de San Marcos, Facultad de Ciencias Administrativas: http://revistasinvestigacion.unmsm.edu.pe/index.php/administrativas/article/view/1170 9

Huamaní, P. L., \& Vargas, S. (2013). Impacto de la Motivación y el liderazgo en el Rendimiento Laboral en una Empresa de Servicios de Lima Metropolitana. Recuperado 
de la Universidad Mayor de San Marcos, Facultad de Ciencias Administrativas: http://revistasinvestigacion.unmsm.edu.pe/index.php/administrativas/article/view/8754

Kaiser, H. F. (1974). An index of factorial simplicity. Psychometrika, 39, 31-36. doi: 10.1007/BF02291575

Kasser, T., \& Ryan, R. M. (1996). Further examining the American dream: Differential correlates of intrinsic and extrinsic goals. Personality and Social Psychology Bulletin, 22, 280-287. doi: 10.1177/0146167296223006

Koestner, R., Ryan, R. M., Bernieri, F., \& Holt, K. (1984). Setting limits on children's behavior: The differential effects of controlling versus informational styles on children's intrinsic motivation and creativity. Journal of Personality, 54, 233-248. doi: 10.1111/j.1467-6494.1984.tb00879.x

Moreau, E., \& Mageau, G. A. (2012). The importance of perceived autonomy support for the psychological health and work satisfaction of health professionals: not only supervisors count, colleagues too! Motivation and Performance, 36, 268-286. doi:10.1007/s11031011-9250-9

Nishimura, T., \& Suzuki, T. (2016). Basic psychological need satisfaction and frustration in Japan: Controlling for the big five personality traits. Japanese Psychological Research, 58, 320-331. doi: 10.1111/jpr.12131

Pelletier, L. G., Fortier, M. S., Vallerand, R. J., \& Brière, N. M. (2001). Associations Among Perceived Autonomy Support, Forms of Self-Regulation, and Persistence: A Prospective Study. Motivation and Emotion, 25(4), 279-306. doi: 10.1023/a:1014805132406

Reeve, J. (2009). Why teachers adopt a controlling motivating style toward students and how they can become more autonomy supportive. Educational Psychologist, 44(3), 159-175. doi: 10.1080/00461520903028990 
Richer, S. F., \& Vallerand, R. J. (1995). Supervisors' interactional styles and subordinates' intrinsic and extrinsic motivation. Journal of Social Psychology, 135(6), 707-722. doi: $10.1080 / 00224545.1995 .9713974$

Ryan, R. M. (1982). Control and information in the intrapersonal sphere: An extension of cognitive evaluation theory. Journal of Personality and Social Psychology, 43, 450-461. doi: 10.1037/0022-3514.43.3.450

Ryan, R. M. (1995). Psychological needs and the facilitation of integrative processes. Journal of Personality, 63, 397-427. doi: 10.1111/j.1467-6494.1995.tb00501.x

Ryan, R. M., \& Connell, J.P. (1989). Perceived locus of causality and internalization: Examining reasons for acting in two domains. Journal of Personality and Social Psychology, 57, 749-761. doi: 10.1037/0022-3514.57.5.749

Ryan, R. M. \& Deci, E. L. (2000). Self-determination theory and the facilitation of intrinsic motivation, social development, and well-being. American Psychologist, 55, 68-78. doi: 10.1037/0003-066X.55.1.68

Ryan, R. M., \& Deci, E. L. (2002). Overview of Self-Determination Theory: An Organismic Dialectical Perspective. En Autores (Eds.), Handbook of Self-Determination Research (pp. 3-33). New York: The University of Rochester Press.

Simms, L.J., Watson, D., \& Doebbeling, B.N. (2002). Confirmatory Factor Analyses of Posttraumatic Stress Symptoms in Deployed and Nondeployed Veterans of the Gulf War. Journal of Abnormal Psychology, 111, 637-647

Slemp, G. R., Kern, M. L., \& Vella-Brodrick, D. A. (2015). Workplace Well-Being: The Role of Job Crafting and Autonomy Support. Psych Well-Being, 5(7). doi: 10.1186/s13612015-0034-y 
Soenens, B., Sierens, E., Vansteenkiste, M., Dochy, F., \& Goossens, L. (2012). Psychologically controlling teaching: Examining outcomes, antecedents, and mediators. Journal of Educational Psychology, 104(1), 108-120. doi:10.1037/a0025742

Spencer, N., (2017). Estilo motivacional del docente, tipos de motivación, autoeficacia, compromiso agente y rendimiento en matemáticas en universitarios. (Tesis de posgrado). Universidad Católica del Perú, Lima, Perú.

Uduji, J. \& Ankeli, M. (2013). Needs for Achievement, Affiliation, and Power: the Possible Sales Manager's Action for Exceptional Salesforce Performance, Research Journal of Finance and Accounting, 4, 96-103. Recuperado de: http://pakacademicsearch.com/pdffiles/ech/519/96103\%20Vol\%204,\%20No\%209\%20(2013).pdf

Van den Broeck, A., Vansteenkiste, M., De Witte, H., \& Lens, W. (2008). Explaining the relationships between job characteristics, burnout and engagement: The role of basic psychological need satisfaction. Work and Stress, 22 (3), 277-294. doi: $10.1080 / 02678370802393672$

Van den Broeck, A., Vansteenkiste, M., De Witte, H., Soenens, B., \& Lens, W. (2010). Capturing autonomy, competence, and relatedness at work: Construction and initial validation of the Work-related Basic Need Satisfaction scale. Journal of Occupational and Organizational Psychology, 83, 981-1002. doi: 10.1348/096317909X481382

Vansteenkiste, M., Niemiec, C. P., \& Soenens, B. (2010). The development of the five minitheories of self-determination theory: An historical overview, emerging trends, and future directions. En T. C. Urdan \& S. Karabenick (Eds.), The Decade Ahead: Theoretical Perspectives on Motivation and Achievement (pp. 105-166). doi: 10.1108

White, R. W. (1959). Motivation Reconsidered: The concept of competence. Psychological Review, 66(5), 297-333. doi: 10.1037/h0040934 
Williams, G. C., Gagne', M., Ryan, R. M., \& Deci, E. L. (2002). Facilitating autonomous motivation for smoking cessation. Health Psychology, 21(1), 40-50. doi: 10.1037//02786133.21.1.40

Zuckerman, M., Porac, J., Lathin, D., \& Deci, E. (1978). On the Importance of selfdetermination for intrinsically-motivated behaviour. Personality and Social Psychology Bulletin, 4, 443-446. doi: 10.1177/014616727800400317 


\section{Anexos}

\section{Consentimiento Informado}

El presente documento es un consentimiento informado a través del cual se le solicita su autorización para participar en la investigación presentada, la cual está siendo llevada a cabo por Tamara Fosca Gagliuffi y Alexia García Hoefken, estudiantes de la Carrera de Psicología Organizacional en la Universidad Peruana de Ciencias Aplicadas (UPC).

Los objetivos de esta investigación consisten en conocer la relación entre las necesidades psicológicas básicas y el estilo de motivación del supervisor; además de conocer la relación entre los estilos de motivación y el bienestar psicológico.

Para fines de este estudio se le aplicará cuatro cuestionarios. Su participación consistirá en responder 93 ítems. Si usted acepta participar en este estudio, se le pedirá que complete los datos del presente consentimiento informado y los cuestionarios que se darán más adelante. Responder a estos cuestionarios le tomará aproximadamente 20 minutos.

Los cuestionarios presentados se responderán de manera anónima.

La información recopilada acerca de usted se tratará en forma confidencial.

Los datos recolectados solo se utilizarán para fines de esta investigación.

Su participación en esta investigación es totalmente voluntaria, es decir, usted puede decidir no continuar en el momento que crea pertinente.

Por favor, si usted decide continuar con esta investigación, responda todas las preguntas sin obviar alguna y con total sinceridad.

En el caso que tenga alguna duda o consulta, podrá contactar a las autoras en las siguientes direcciones electrónicas: u610323@upc.edu.pe (Alexia García Hoefken); u710156@upc.edu.pe (Tamara Fosca Gagliuffi). 
La firma de este documento significa que usted está aceptando responder las preguntas que se encuentran en los cuestionarios.

Muchas gracias por su participación.

FIRMA DEL PARTICIPANTE

\section{Datos Demográficos}

A continuación encontrará algunas preguntas sobre su vida personal y laboral. En ese sentido, solicitamos su colaboración para responder a los siguientes enunciados, recordándole que la encuesta es completamente anónima y confidencial. No existen respuestas buenas o malas, sólo deseamos conocer su opinión acerca de lo que se le pregunta.

1. Sexo: (1) Femenino $\quad$ (2) Masculino

2. Edad:

3. Lugar de nacimiento: (1) Lima $\quad$ (2) Provincia $\quad$ (3) Extranjero

Especifique:

4. Estado civil:
(1) Soltero
(3) Viudo
(5) Separado
(2) Casado
(4) Divorciado
(6) Conviviente

5. Número de hijos: $0 \begin{array}{lllllllll}0 & 1 & 2 & 3 & 4 & 5 & 6 & 7 & 8\end{array}$

6. Grado máximo de instrucción alcanzado:
(1) Primaria incompleta
(5) Universitario incompleta
(2) Primaria completa
(6) Universitario completa
(3) Secundaria incompleta
(7) Técnica completa
(4) Secundaria completa
(8) Técnica incompleta
(9) Postgrado (Maestría o Doctorado)

7. Nombre de Carrera Universitaria que estudió:

8. Número de capacitaciones y talleres recibidos en el año 2011 y 2012: $0 \begin{array}{lllllll}0 & 1 & 2 & 3 & 4 & 5 & 6\end{array}$ Especificar tema:

9. Posición que desempeña:
(1) Asistente
(3) Jefe (a)
(5) Gerente
(2) Analista
(4) Supervisor /Gestor
(6) Otro

10. Tiempo que ha desempañado dicha posición en la empresa:
(1) Menos de 1 año
(3) Entre 3 a 5 años y 11 meses 
(2) Entre 1 a 2 años y 11 meses

(4) Más de 6 años Especifique:

11. Tiempo que ha laborado en la empresa:
(1) Menos de 1 año
(3) Entre 3 a 5 años y 11 meses
(2) Entre 1 a 2 años y 11 meses
(4) Más de 6 años Especifique:

12. Años de experiencia en general:
(1) Menos de 1 año
(3) Entre 3 a 5 años y 11 meses
(2) Entre 1 a 2 años y 11 meses
(4) Más de 6 años Especifique:

13. Número de personas a su cargo (Empleo Actual):
(1) Ninguna
(3) De 3 a 5
(5) De 11 a 20
(7) De 31 a 50
(2) De 1 a 2
(4) De 6 a 10 (6) de 21 a 30
(8) Más de 50 Especifique:

14. Tiempo dirigiendo su equipo actual:
(1) Menos de 1 año
(3) Entre 3 a 5 años y 11 meses
(2) Entre 1 a 2 años y 11 meses
(4) Más de 6 años Especifique:

15. Área de trabajo:
(1) Comercial/Ventas
(5) Sistemas
(2) Marketing
(6) Recursos Humanos
(3) Administración
(7) Contabilidad
(4) Finanzas
(8) Otro:

16. Rubro de la empresa:

*Por favor, verifique que haya contestado a todos los enunciados. 
